\title{
An Acid-Free Anionic Oxoborane Isoelectronic with Carbonyl: Facile Access and Transfer of a Terminal $B=0$ Double Bond
}

Ying Kai Loh, Kieran Porteous, M. Ángeles Fuentes, Dinh Cao Huan Do, Jamie Hicks, Simon Aldridge*

Inorganic Chemistry Laboratory, Department of Chemistry, University of Oxford, South Parks Road, Oxford, OX1 3QR UK

Table of Contents

1. General considerations and starting material preparations

2. Synthetic, spectroscopic and analytical data - 1, 2, 3, 4, 5, $(\mathrm{HCDippN})_{2} \mathrm{BH},(\mathrm{HCDippN})_{2} \mathrm{BCl},(\mathrm{OC}(\mathrm{p}-\mathrm{Tol}) \mathrm{N})_{2} \mathrm{CO}$

3. NMR spectra of compounds $\mathbf{1}, \mathbf{2}, \mathbf{3}, \mathbf{4}$ and 5

4. X-ray crystallographic studies

5. Computational studies

6. References 


\section{General considerations and starting material preparation}

All manipulations were carried out using standard Schlenk line or dry-box techniques under an atmosphere of argon or dinitrogen. Solvents were degassed by sparging with argon and dried by passing through a column of the appropriate drying agent. NMR spectra were measured in benzene- $d_{6}$ (which was dried over potassium), with the solvent then being distilled under reduced pressure and stored under argon in Teflon valve ampoules. NMR samples were prepared under argon in $5 \mathrm{~mm}$ Wilmad 507-PP tubes fitted with J. Young Teflon valves. ${ }^{1} \mathrm{H},{ }^{13} \mathrm{C}\left\{{ }^{1} \mathrm{H}\right\},{ }^{11} \mathrm{~B}\left\{{ }^{1} \mathrm{H}\right\}$ and ${ }^{19} \mathrm{~F}\left\{{ }^{1} \mathrm{H}\right\}$ NMR spectra were recorded on Bruker Avance III HD nanobay $400 \mathrm{MHz}$ or Bruker Avance $500 \mathrm{MHz}$ spectrometer at ambient temperature and referenced internally to residual protio-solvent $\left({ }^{1} \mathrm{H}\right)$ or solvent $\left({ }^{13} \mathrm{C}\right)$ resonances and are reported relative to tetramethylsilane $(\delta=0 \mathrm{ppm}) .{ }^{19} \mathrm{~F}$ resonances are referenced externally to $\mathrm{CFCl}_{3}$. Assignments were confirmed using two-dimensional ${ }^{1} \mathrm{H}-{ }^{1} \mathrm{H}$ and ${ }^{13} \mathrm{C}-{ }^{1} \mathrm{H}$ NMR correlation experiments. Chemical shifts are quoted in $\delta(\mathrm{ppm})$ and coupling constants in Hz. Elemental analyses were carried out by London Metropolitan University. $\left\{\mathrm{K}\left[(\mathrm{HCDippN})_{2} \mathrm{BO}\right]\right\}_{2}$ was prepared by literature method. ${ }^{1}$ All other reagents were used as received. 
Preparation of [K(2.2.2-crypt)][(HCDippN $)_{2}$ BO] [1]. To a mixture of $\left\{\mathrm{K}\left[(\mathrm{HCDippN})_{2} \mathrm{BO}\right]\right\}_{2}(200 \mathrm{mg}, 0.23 \mathrm{mmol})$ and [2.2.2]cryptand $(170 \mathrm{mg}, 0.45 \mathrm{mmol})$ was added toluene $(5 \mathrm{~mL})$ and the reaction mixture stirred for $5 \mathrm{~min}$ at room temperature to form a red solution. Volatiles were removed under vacuum to yield $[\mathrm{K}(2.2 .2$-crypt $)]\left[(\mathrm{HCDippN})_{2} \mathrm{BO}\right][1]$ (342 mg, 93\% yield) as a red powder. Single crystals suitable for X-ray crystallography were obtained by slow evaporation of a concentrated solution of $[\mathrm{K}(2.2 .2$-crypt $)]\left[(\mathrm{HCDippN})_{2} \mathrm{BO}\right][1]$ in benzene at room temperature. ${ }^{1} \mathrm{H} \mathrm{NMR}$ $\left(500 \mathrm{MHz}, \mathrm{C}_{6} \mathrm{D}_{6}, 297 \mathrm{~K}\right): \delta=1.48\left(\mathrm{~d},{ }^{3} J_{\mathrm{HH}}=6.0 \mathrm{~Hz}, 12 \mathrm{H}, \mathrm{CH}\left(\mathrm{CH}_{3}\right)_{2}\right), 1.50\left(\mathrm{~d},{ }^{3} J_{\mathrm{HH}}=6.9 \mathrm{~Hz}, 12 \mathrm{H}, \mathrm{CH}\left(\mathrm{CH}_{3}\right)_{2}\right), 2.28(\mathrm{t}$, ${ }^{3} J_{\mathrm{HH}}=4.8 \mathrm{~Hz}, 12 \mathrm{H}$, crypt-CH $\left.\mathrm{C}_{2}\right), 3.11\left(\mathrm{t},{ }^{3} J_{\mathrm{HH}}=4.8 \mathrm{~Hz}, 12 \mathrm{H}\right.$, crypt-CH$), 3.16\left(\mathrm{~s}, 12 \mathrm{H}\right.$, crypt-CH$\left.{ }_{2}\right), 4.09\left(\mathrm{sept},{ }^{3} J_{\mathrm{HH}}=6.7\right.$ $\left.\mathrm{Hz}, 4 \mathrm{H}, \mathrm{CH}\left(\mathrm{CH}_{3}\right)_{2}\right), 6.26(\mathrm{~s}, 2 \mathrm{H}, \mathrm{NCH}), 7.21-7.28$ (m, 6H, Dipp-CH); ${ }^{13} \mathrm{C}\left\{{ }^{1} \mathrm{H}\right\}$ NMR $\left(126 \mathrm{MHz}, \mathrm{C}_{6} \mathrm{D}_{6}\right): \delta=24.3$ $\left(\mathrm{CH}\left(\mathrm{CH}_{3}\right)_{2}\right), 25.1\left(\mathrm{CH}\left(\mathrm{CH}_{3}\right)_{2}\right), 28.7\left(\mathrm{CH}\left(\mathrm{CH}_{3}\right)_{2}\right), 56.1$ (crypt- $\left.\mathrm{CH}_{2}\right), 68.6\left(\right.$ crypt- $\left.\mathrm{CH}_{2}\right), 70.2$ (crypt- $\left.\mathrm{CH}_{2}\right), 115.7(\mathrm{NCH})$, 122.6 (Dipp- $m-C H), 124.4$ (Dipp-p-CH), 146.2 (Dipp-i-C), 148.2 (Dipp-o-C); ${ }^{11} \mathrm{~B}\left\{{ }^{1} \mathrm{H}\right\}$ NMR $\left(128 \mathrm{MHz}, \mathrm{C}_{6} \mathrm{D}_{6}\right): \delta=20.7$; anal. calc. for $\mathrm{C}_{44} \mathrm{H}_{72} \mathrm{BKN}_{4} \mathrm{O}_{7}$ : C 64.53\%, H 8.86\%, N 6.84\%, found: C 64.64\%, H 9.02\%, N 6.72\%.

Preparation of [K(2.2.2-crypt)][(HCDippN $)_{2}$ BS] [2]. To a solution of [K(2.2.2-crypt)][(HCDippN $\left.)_{2} \mathrm{BO}\right]$ [1] $(50 \mathrm{mg}, 0.06$ $\mathrm{mmol})$ in benzene $(0.3 \mathrm{~mL})$ was added $\mathrm{CS}_{2}(0.05 \mathrm{~mL}, 0.83 \mathrm{mmol})$ at room temperature. Volatiles were removed under vacuum to yield $[\mathrm{K}(2.2 .2$-crypt $)]\left[(\mathrm{HCDippN})_{2} \mathrm{BS}\right]$ [2] $(51 \mathrm{mg}, 100 \%$ yield $)$ as a red powder. Single crystals suitable for $\mathrm{X}$-ray crystallography were obtained by slow evaporation of a concentrated solution of $[\mathrm{K}(2$.2.2-crypt $)]\left[(\mathrm{HCDippN})_{2} \mathrm{BS}\right]$ [2] in benzene at room temperature. ${ }^{1} \mathrm{H}$ NMR $\left(500 \mathrm{MHz}, \mathrm{C}_{6} \mathrm{D}_{6}, 297 \mathrm{~K}\right): \delta=1.50\left(\mathrm{~d},{ }^{3} J_{\mathrm{HH}}=7.0 \mathrm{~Hz}, 12 \mathrm{H}, \mathrm{CH}\left(\mathrm{CH}_{3}\right)_{2}\right), 1.60$ $\left.\left(\mathrm{d},{ }^{3} \mathrm{~J}_{\mathrm{HH}}=6.9 \mathrm{~Hz}, 12 \mathrm{H}, \mathrm{CH}\left(\mathrm{CH}_{3}\right)_{2}\right), 2.23\left(\mathrm{t},{ }^{3} \mathrm{~J}_{\mathrm{HH}}=4.6 \mathrm{~Hz}, 12 \mathrm{H}, \text { crypt-CH}\right)_{2}\right), 3.24\left(\mathrm{t},{ }^{3} J_{\mathrm{HH}}=4.6 \mathrm{~Hz}, 12 \mathrm{H}\right.$, crypt-CH$)$, $3.28\left(\mathrm{~s}, 12 \mathrm{H}\right.$, crypt-CH $\left.\mathrm{CH}_{2}\right), 4.00\left(\mathrm{sept},{ }^{3} J_{\mathrm{HH}}=6.9 \mathrm{~Hz}, 4 \mathrm{H}, \mathrm{CH}\left(\mathrm{CH}_{3}\right)_{2}\right), 6.47$ (s, 2H, NCH), 7.27-7.32 (m, 6H, Dipp-CH);

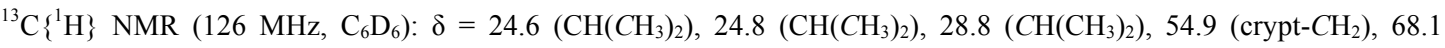
(crypt- $\mathrm{CH}_{2}$ ), 70.6 (crypt- $\mathrm{CH}_{2}$ ), 117.4 ( $\mathrm{NCH}$ ), 122.5 (Dipp-m-CH), 125.3 (Dipp-p-CH), 145.1 (Dipp-i-C), 148.0 (Dipp-oC); ${ }^{11} \mathrm{~B}\left\{{ }^{1} \mathrm{H}\right\}$ NMR $\left(128 \mathrm{MHz}, \mathrm{C}_{6} \mathrm{D}_{6}\right): \delta=33.1$; anal. calc. for $\mathrm{C}_{44} \mathrm{H}_{72} \mathrm{BKN}_{4} \mathrm{O}_{6} \mathrm{~S}: \mathrm{C} 63.29 \%, \mathrm{H} 8.69 \%, \mathrm{~N} 6.71 \%$, found: $\mathrm{C}$ $63.43 \%, \mathrm{H} 8.84 \%$, N $6.67 \%$.

Preparation of $\left\{\boldsymbol{K}\left[(\boldsymbol{H C D i p p N})_{2} \boldsymbol{B S}\right]\right\}_{4}$ [3]. To a solution of $\left\{\mathrm{K}\left[(\mathrm{HCDippN})_{2} \mathrm{BO}\right]\right\}_{2}(200 \mathrm{mg}, 0.23 \mathrm{mmol})$ in toluene $(2 \mathrm{~mL})$ was added $\mathrm{CS}_{2}(0.1 \mathrm{~mL}, 1.66 \mathrm{mmol})$ at room temperature. The solution was concentrated until the precipitation of solid, and subsequently washed with additional toluene $(1 \mathrm{~mL})$. Volatiles were removed under vacuum to yield $\left\{\mathrm{K}\left[(\mathrm{HCDippN})_{2} \mathrm{BS}\right]\right\}_{4}[3]$ (104 mg, 51\% yield) as a pale yellow powder. Single crystals suitable for X-ray crystallography were obtained by slow evaporation of a concentrated solution of $\left\{\mathrm{K}\left[(\mathrm{HCDippN})_{2} \mathrm{BS}\right]\right\}_{4}[3]$ in benzene at room temperature. ${ }^{1} \mathrm{H}$ NMR $\left(500 \mathrm{MHz}, \mathrm{C}_{6} \mathrm{D}_{6}, 297 \mathrm{~K}\right): \delta=1.28\left(\mathrm{~d},{ }^{3} \mathrm{~J}_{\mathrm{HH}}=6.9 \mathrm{~Hz}, 24 \mathrm{H}, \mathrm{CH}\left(\mathrm{CH}_{3}\right)_{2}\right), 3.46(\mathrm{bs}, 4 \mathrm{H}$, $\left.\mathrm{C} H\left(\mathrm{CH}_{3}\right)_{2}\right), 6.13$ (s, 2H, NCH), 7.13 (s, 6H, Dipp-CH); ${ }^{13} \mathrm{C}\left\{{ }^{1} \mathrm{H}\right\}$ NMR $\left(126 \mathrm{MHz}, \mathrm{C}_{6} \mathrm{D}_{6}\right): \delta=24.4\left(\mathrm{CH}\left(\mathrm{CH}_{3}\right)_{2}\right), 28.5$ $\left(\mathrm{CH}\left(\mathrm{CH}_{3}\right)_{2}\right), 118.0(\mathrm{NCH}), 123.6$ (Dipp-m-CH), 127.0 (Dipp-p-CH), 143.0 (Dipp-i-C), 148.1 (Dipp-o-C); ${ }^{11} \mathrm{~B}\left\{{ }^{1} \mathrm{H}\right\} \mathrm{NMR}$ $\left(128 \mathrm{MHz}, \mathrm{C}_{6} \mathrm{D}_{6}\right): \delta=32.1$; anal. calc. for $\mathrm{C}_{104} \mathrm{H}_{144} \mathrm{~B} 4 \mathrm{~K} 4 \mathrm{~N}_{8} \mathrm{~S}_{4}: \mathrm{C} 68.10 \%, \mathrm{H} 7.91 \%, \mathrm{~N} 6.11 \%$, found: $\mathrm{C} 67.97 \%, \mathrm{H} 7.86 \%$, N $6.01 \%$.

Preparation of [(HCDippN $)_{2} \boldsymbol{B}\left(\boldsymbol{N C}_{5} \boldsymbol{H}_{5}\right)$ ][OTf] [4]. To a solution of [K(2.2.2-crypt)][(HCDippN $\left.)_{2} \mathrm{BO}\right]$ [1] (50 mg, 0.06 $\mathrm{mmol})$ in benzene $(5 \mathrm{~mL})$ was added $\mathrm{Tf}_{2} \mathrm{O}$ solution $0.02 \mathrm{M}$ in benzene $(0.3 \mathrm{~mL}, 0.06 \mathrm{mmol})$ and pyridine $(1 \mathrm{~mL})$ at room temperature. Volatiles were removed under vacuum. To the residue was added diethyl ether $(5 \mathrm{~mL})$ and the mixture filtered. The filtrate was dried under vacuum to yield [( $\left.\mathrm{HCDippN})_{2} \mathrm{~B}\left(\mathrm{NC}_{5} \mathrm{H}_{5}\right)\right][\mathrm{OTf}]$ [4] $(19 \mathrm{mg}, 51 \%$ yield $)$ as a bright yellow powder. Single crystals suitable for X-ray crystallography were obtained by slow evaporation of a concentrated solution of $\left[(\mathrm{HCDippN})_{2} \mathrm{~B}\left(\mathrm{NC}_{5} \mathrm{H}_{5}\right)\right][\mathrm{OTf}]$ [4] in 1,2-difluorobenzene at room temperature. ${ }^{1} \mathrm{H} \mathrm{NMR}\left(500 \mathrm{MHz}, \mathrm{CD}_{2} \mathrm{Cl}_{2}\right.$, $297 \mathrm{~K}): \delta=1.01\left(\mathrm{~d},{ }^{3} J_{\mathrm{HH}}=7.0 \mathrm{~Hz}, 12 \mathrm{H}, \mathrm{CH}\left(\mathrm{CH}_{3}\right)_{2}\right), 1.25\left(\mathrm{~d},{ }^{3} J_{\mathrm{HH}}=6.8 \mathrm{~Hz}, 12 \mathrm{H}, \mathrm{CH}\left(\mathrm{CH}_{3}\right)_{2}\right), 2.85\left(\mathrm{sept},{ }^{3} J_{\mathrm{HH}}=6.9 \mathrm{~Hz}\right.$, $\left.4 \mathrm{H}, \mathrm{CH}\left(\mathrm{CH}_{3}\right)_{2}\right), 6.56(\mathrm{~s}, 2 \mathrm{H}, \mathrm{NCH}), 7.36\left(\mathrm{~d},{ }^{3} J_{\mathrm{HH}}=7.8 \mathrm{~Hz}, 4 \mathrm{H}, \mathrm{Dipp}-m-\mathrm{CH}\right), 7.50\left(\mathrm{t},{ }^{3} J_{\mathrm{HH}}=7.8 \mathrm{~Hz}, 2 \mathrm{H}\right.$, Dipp- $\left.p-\mathrm{CH}\right)$, 7.94-7.97 (m, 2H, Рy-m-CH), 8.03-8.04 (m, 2H, Py-o-CH), 8.70-8.73 (m, 1H, Рy-p-CH); ${ }^{13} \mathrm{C}\left\{{ }^{1} \mathrm{H}\right\}$ NMR (126 MHz,

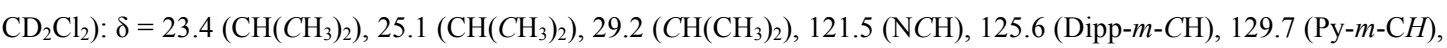


130.3 (Dipp-p-CH), 134.2 (Dipp-i-C), 143.8 (Py-o-CH), 145.4 (Dipp-o-C), 149.6 (Py-p-CH); ${ }^{11} \mathrm{~B}\left\{{ }^{1} \mathrm{H}\right\}$ NMR (128 MHz, $\left.\mathrm{CD}_{2} \mathrm{Cl}_{2}\right): \delta=20.4 ;{ }^{19} \mathrm{~F}\left\{{ }^{1} \mathrm{H}\right\} \mathrm{NMR}\left(377 \mathrm{MHz}, \mathrm{CD}_{2} \mathrm{Cl}_{2}\right): \delta=-79.0$; anal. calc. for $\mathrm{C}_{32} \mathrm{H}_{40} \mathrm{BF}_{3} \mathrm{~N}_{3} \mathrm{O}_{3} \mathrm{~S}: \mathrm{C} 62.54 \%, \mathrm{H} 6.56 \%, \mathrm{~N}$ $6.84 \%$, found: C $62.35 \%, \mathrm{H} 6.71 \%$, N $6.75 \%$.

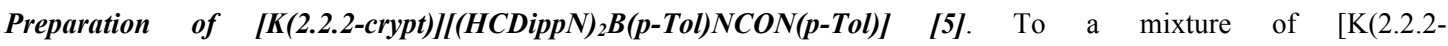
crypt)][(HCDippN $)_{2} \mathrm{BO}$ [1] (100 mg, $\left.0.12 \mathrm{mmol}\right)$ and $(p$-Tol)NCN( $p$-Tol) $(27 \mathrm{mg}, 0.12 \mathrm{mmol})$ was added benzene $(0.5$ $\mathrm{mL})$ and the reaction mixture agitated at room temperature to form a red solution. Single crystals of $[\mathrm{K}(2.2 .2-$ crypt)][(HCDippN $)_{2} \mathrm{~B}(p$-Tol)NCON( $p$-Tol)] [5] (21 mg, 17\% yield) suitable for X-ray crystallography were obtained from a concentrated solution in tetrahydrofuran at room temperature. ${ }^{1} \mathrm{H}$ NMR $\left(400 \mathrm{MHz}, \mathrm{C}_{6} \mathrm{D}_{6}, 297 \mathrm{~K}\right): \delta=1.31\left(\mathrm{~d},{ }^{3} J_{\mathrm{HH}}\right.$ $\left.=6.8 \mathrm{~Hz}, 12 \mathrm{H}, \mathrm{CH}\left(\mathrm{CH}_{3}\right)_{2}\right), 1.45\left(\mathrm{~d},{ }^{3} J_{\mathrm{HH}}=6.8 \mathrm{~Hz}, 12 \mathrm{H}, \mathrm{CH}\left(\mathrm{CH}_{3}\right)_{2}\right), 2.00\left(\mathrm{t},{ }^{3} J_{\mathrm{HH}}=4.6 \mathrm{~Hz}, 12 \mathrm{H}\right.$, crypt-CH$), 2.32(\mathrm{~s}, 6 \mathrm{H}$, $p$-Tol- $\left.\mathrm{CH}_{3}\right), 2.98\left(\mathrm{t},{ }^{3} J_{\mathrm{HH}}=4.6 \mathrm{~Hz}, 12 \mathrm{H}\right.$, crypt-CH$), 3.06\left(\mathrm{~s}, 12 \mathrm{H}\right.$, crypt- $\left.\mathrm{CH}_{2}\right), 4.32-4.38\left(\mathrm{~m}, 4 \mathrm{H}, \mathrm{CH}\left(\mathrm{CH}_{3}\right)_{2}\right), 6.39(\mathrm{~s}, 2 \mathrm{H}$, $\mathrm{NCH}), 7.04\left(\mathrm{~d},{ }^{3} J_{\mathrm{HH}}=8.2 \mathrm{~Hz}, 4 \mathrm{H}, p-\mathrm{Tol}-\mathrm{C} H\right), 7.18-7.25(\mathrm{~m}, 6 \mathrm{H}, \mathrm{Dipp}-\mathrm{CH}), 7.64\left(\mathrm{~d},{ }^{3} J_{\mathrm{HH}}=7.9 \mathrm{~Hz}, 4 \mathrm{H}, p-\mathrm{Tol}-\mathrm{CH}\right)$; ${ }^{13} \mathrm{C}\left\{{ }^{1} \mathrm{H}\right\}$ NMR (126 MHz, $\left.\mathrm{C}_{6} \mathrm{D}_{6}\right): \delta=21.4\left(p-\mathrm{Tol}-\mathrm{CH}_{3}\right), 23.9\left(\mathrm{CH}\left(\mathrm{CH}_{3}\right)_{2}\right), 27.4\left(\mathrm{CH}\left(\mathrm{CH}_{3}\right)_{2}\right), 28.4\left(\mathrm{CH}_{(}\left(\mathrm{CH}_{3}\right)_{2}\right), 54.1$ (crypt- $\mathrm{CH}_{2}$ ), 67.6 (crypt- $\mathrm{CH}_{2}$ ), 70.4 (crypt- $\left.\mathrm{CH}_{2}\right), 118.7$ (NCH), 123.3 (Dipp-m- $\left.\mathrm{CH}\right), 124.0$ (p-Tol- $\left.\mathrm{CH}\right), 125.1$ (p-Tol-C), 125.7 (Dipp-p-CH), 143.0 (p-Tol-C), 147.8 (Dipp-i-C), $149.0\left(\right.$ Dipp-o-C), $162.4(\mathrm{~N} C(\mathrm{O}) \mathrm{N}) ;{ }^{11} \mathrm{~B}\left\{{ }^{1} \mathrm{H}\right\}$ NMR $(128 \mathrm{MHz}$, $\left.\mathrm{C}_{6} \mathrm{D}_{6}\right): \delta=17.6$; anal. calc. for $\mathrm{C}_{59} \mathrm{H}_{86} \mathrm{BKN}_{6} \mathrm{O}_{7}$ : C $68.06 \%, \mathrm{H} 8.33 \%$, N 8.07\%, found: $\mathrm{C} 67.76 \%, \mathrm{H} 8.24 \%$, N 7.97\%.

Preparation of (HCDippN $)_{2} \boldsymbol{B H}$. To a mixture of [K(2.2.2-crypt)][(HCDippN $)_{2} \mathrm{BO}$ ] [1] (100 mg, $\left.0.12 \mathrm{mmol}\right)$ and $\mathrm{Me}_{2} \mathrm{HN}_{\mathrm{BH}}(15 \mathrm{mg}, 0.25 \mathrm{mmol})$ was added toluene $(5 \mathrm{~mL})$ and stirred at $80{ }^{\circ} \mathrm{C}$ overnight to form a red solution. Volatiles were removed under vacuum. To the residue was added $n$-hexane $(10 \mathrm{~mL})$ and the mixture filtered. The filtrate was dried under vacuum to yield ( $\mathrm{HCDippN})_{2} \mathrm{BH}(12 \mathrm{mg}, 26 \%$ yield) as a white powder. All spectroscopic data matches previously reported literature values. ${ }^{2}$

Preparation of (HCDippN $)_{2} \boldsymbol{B C l}$. To a solution of [K(2.2.2-crypt)][(HCDippN $)_{2} \mathrm{BO}$ ] [1] (100 $\left.\mathrm{mg}, 0.12 \mathrm{mmol}\right)$ in benzene $(5 \mathrm{~mL})$ was added $\mathrm{POCl}_{3}(0.60 \mathrm{~mL}$ of a $0.02 \mathrm{M}$ solution in benzene, $0.12 \mathrm{mmol})$ to form a yellow solution. Volatiles were removed under vacuum. To the residue was added $n$-hexane $(5 \mathrm{~mL})$ and the mixture filtered. The filtrate was dried under vacuum to yield $(\mathrm{HCDippN})_{2} \mathrm{BCl}(39 \mathrm{mg}, 76 \%$ yield) as a yellow powder. All spectroscopic data matches previously reported literature values. ${ }^{2}$

Preparation of (OC(p-Tol)N $)_{2} \mathrm{CO}$ and $(\mathrm{HCDippN})_{2} \mathbf{B C l}$. To a mixture of $[\mathrm{K}(2.2 .2$-crypt $)]\left[(\mathrm{HCDippN})_{2} \mathrm{BO}\right][\mathbf{1}](100 \mathrm{mg}$, $0.12 \mathrm{mmol})$ and $(p$-Tol $) \mathrm{NCN}(p$-Tol $)(27 \mathrm{mg}, 0.12 \mathrm{mmol})$ was added benzene $(10 \mathrm{~mL})$ and the reaction mixture agitated for $5 \mathrm{~min}$ at room temperature to form a red solution. Oxalyl chloride $(0.10 \mathrm{~mL}, 1.16 \mathrm{mmol})$ was added and volatiles were removed under vacuum. The solid was redissolved in benzene $(10 \mathrm{~mL})$ and stirred at $80{ }^{\circ} \mathrm{C}$ for $1 \mathrm{~h}$ to form a brown solution. Volatiles were removed under vacuum. To the residue was added $n$-hexane $(10 \mathrm{~mL})$ and the mixture filtered. The filtrate was dried under vacuum to yield $(\mathrm{HCDippN})_{2} \mathrm{BCl}(49 \mathrm{mg}, 95 \%$ yield $)$ as a red powder. To the residue was added diethyl ether $(10 \mathrm{~mL})$ and the mixture filtered. The filtrate was dried under vacuum to yield $(\mathrm{OC}(p-\mathrm{Tol}) \mathrm{N})_{2} \mathrm{CO}(17$ $\mathrm{mg}, 48 \%$ yield) as a red powder. All spectroscopic data matches previously reported literature values. ${ }^{2,3}$ 


\section{NMR spectra}

[K(2.2.2-crypt)][(HCDippN)2BO] [1]

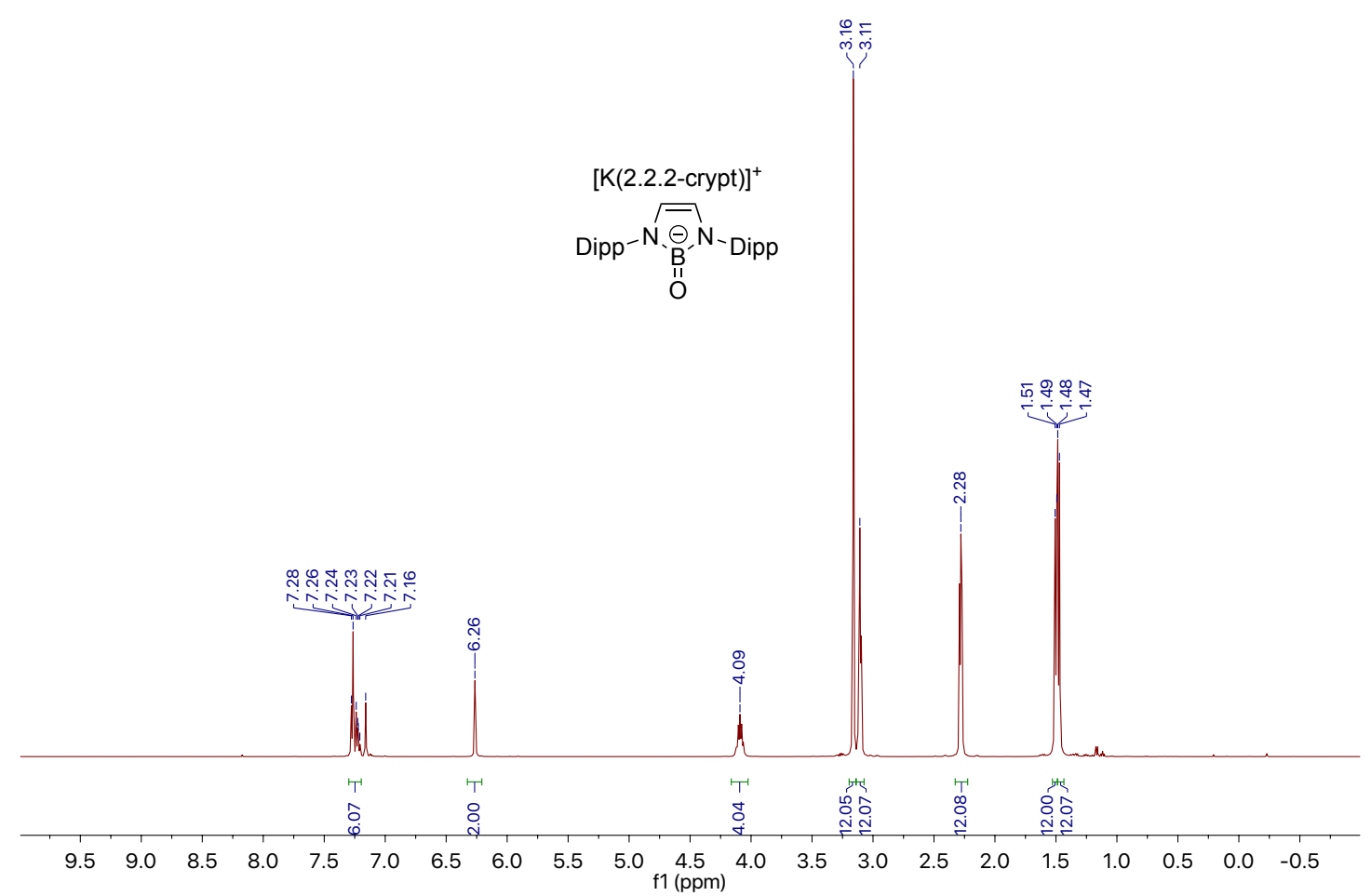

Figure S1. ${ }^{1} \mathrm{H}$ NMR spectrum of 1 . 


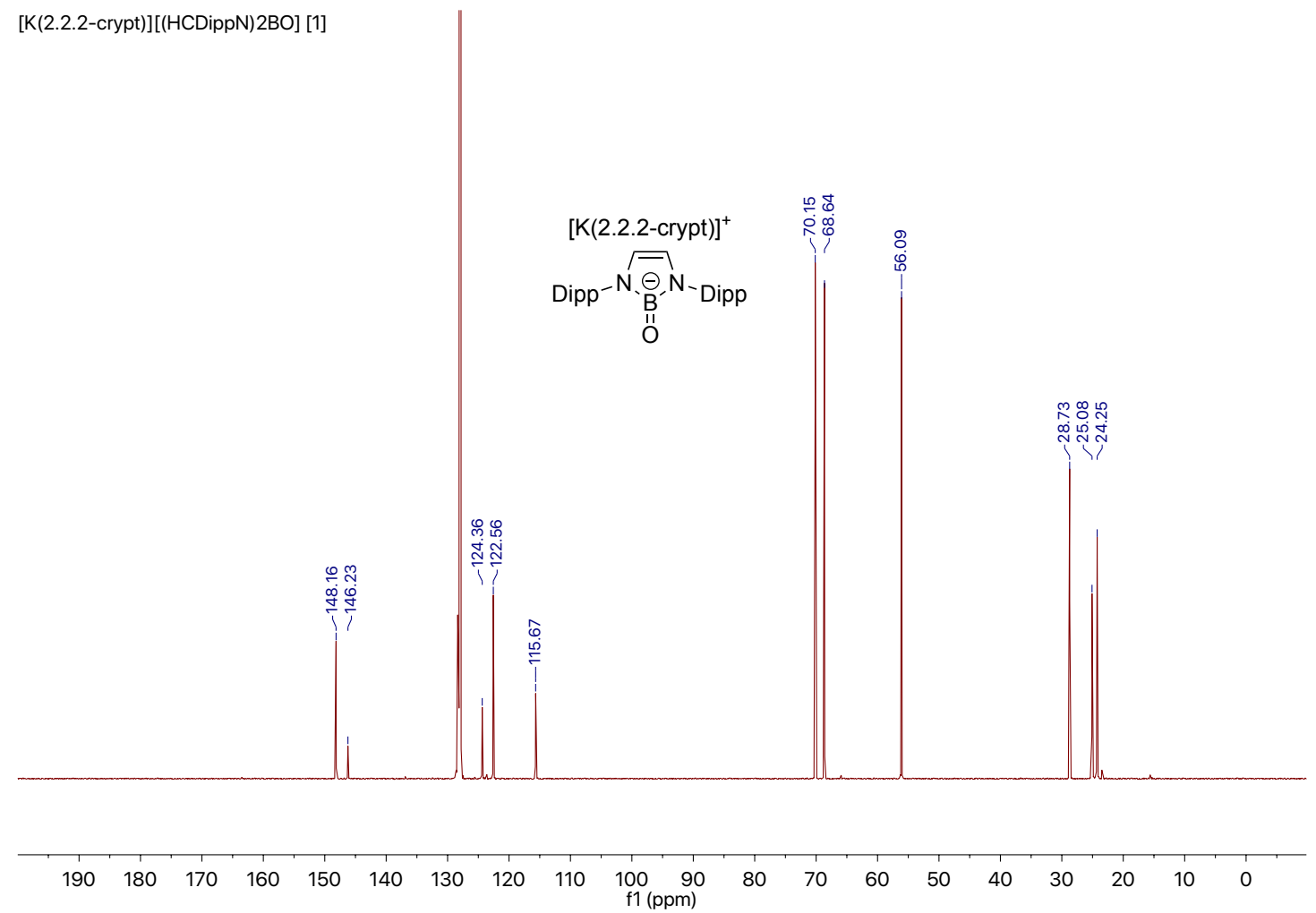

Figure S2. ${ }^{13} \mathrm{C}\left\{{ }^{1} \mathrm{H}\right\}$ NMR spectrum of $\mathbf{1}$.

[K(2.2.2-crypt)][(HCDippN)2BO] [1]
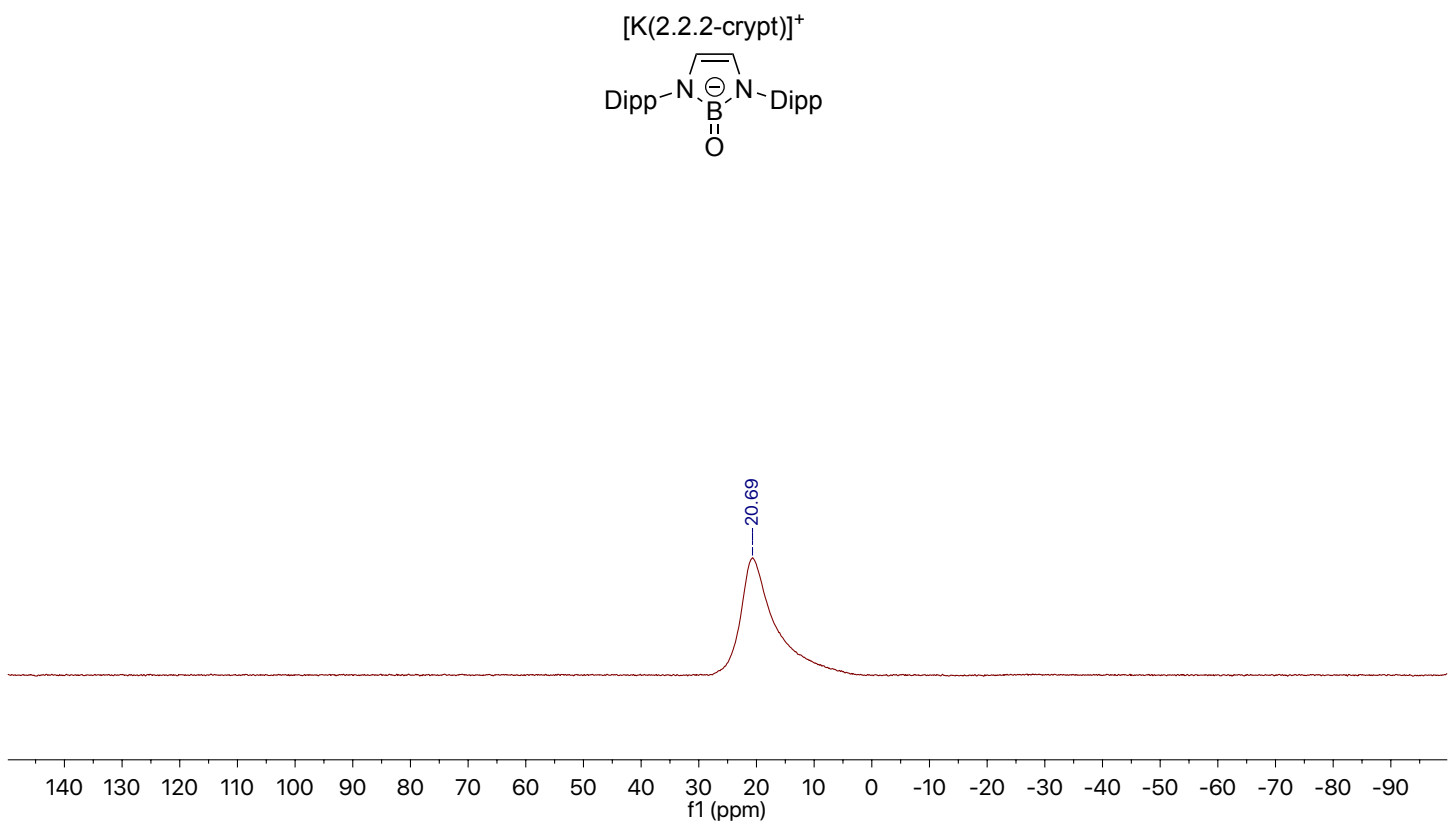

Figure S3. ${ }^{11} \mathrm{~B}\left\{{ }^{1} \mathrm{H}\right\}$ NMR spectrum of $\mathbf{1}$. 


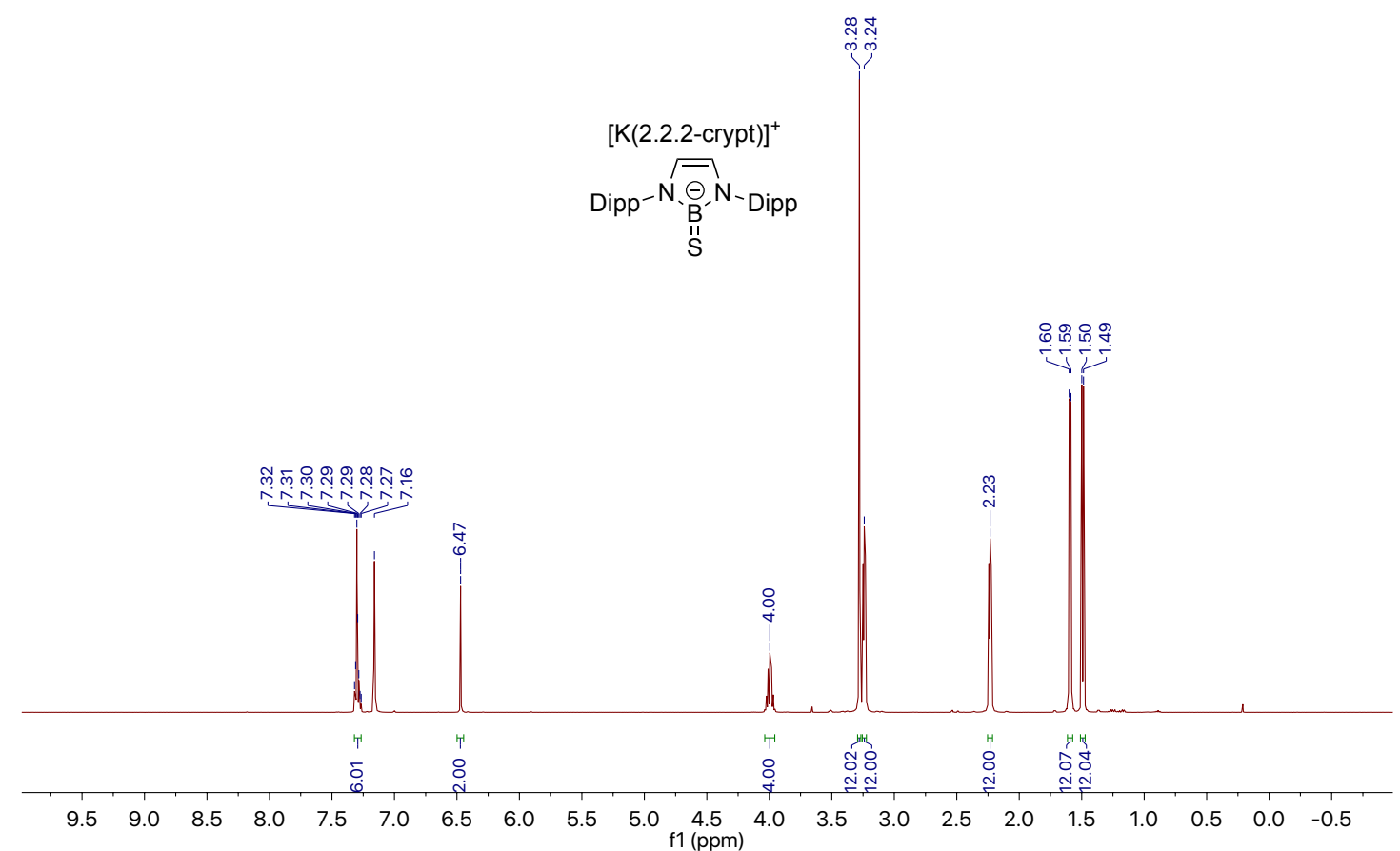

Figure S4. ${ }^{1} \mathrm{H}$ NMR spectrum of 2.

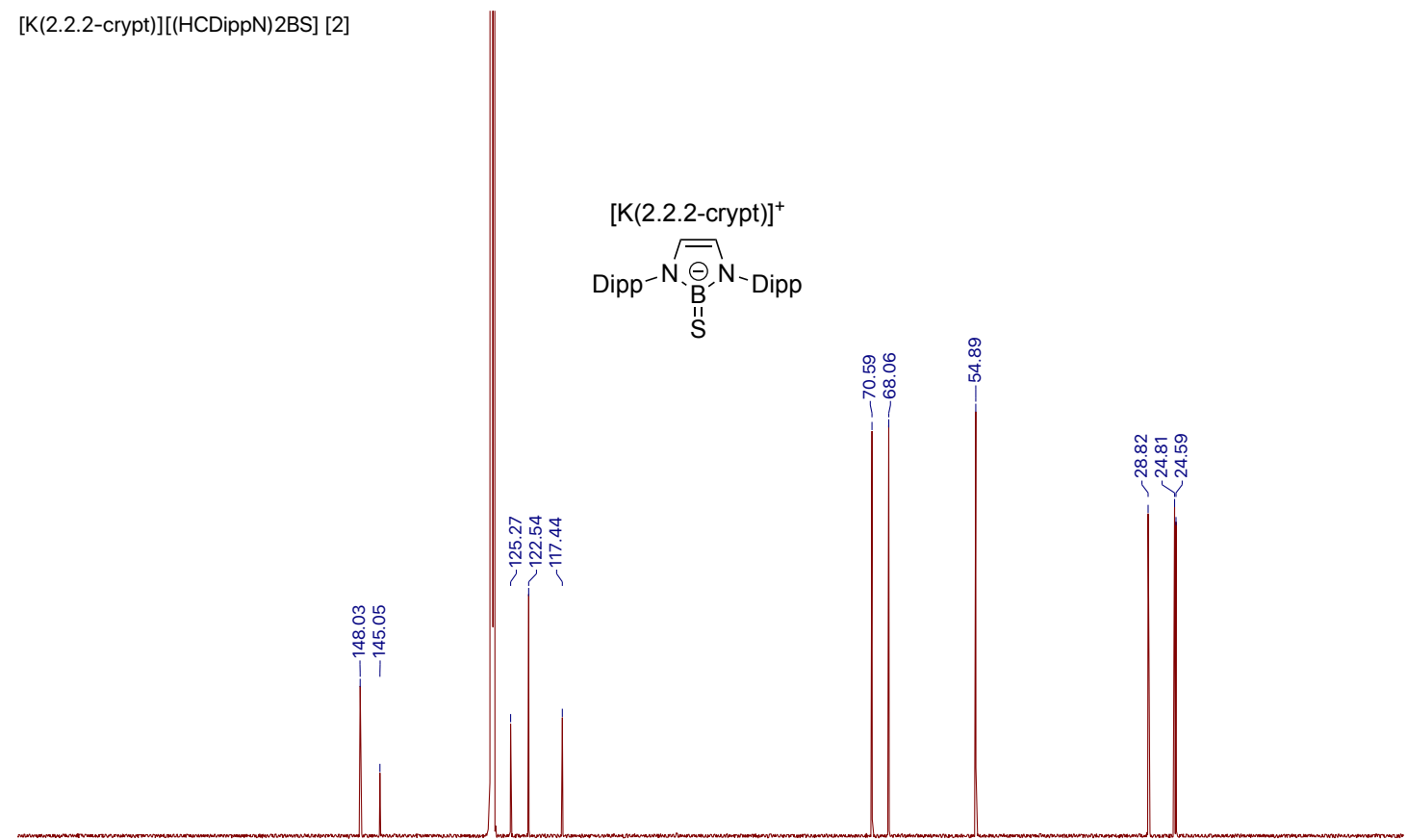

$\begin{array}{lllllllllll}190 & 180 & 170 & 160 & 150 & 140 & 130 & 120 & 110 & \begin{array}{c}100 \\ \mathrm{f} 1(\mathrm{ppm})\end{array}\end{array}$

Figure S5. ${ }^{13} \mathrm{C}\left\{{ }^{1} \mathrm{H}\right\}$ NMR spectrum of 2 . 
[K(2.2.2-crypt)][(HCDippN)2BS] [2]

$$
[\mathrm{K}(2.2 .2-\mathrm{crypt})]^{+}
$$

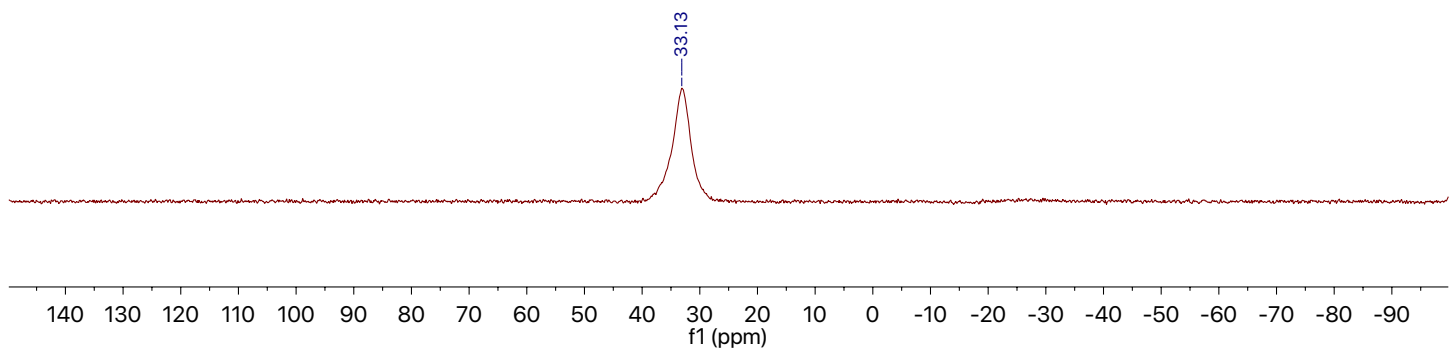

Figure S6. ${ }^{11} \mathrm{~B}\left\{{ }^{1} \mathrm{H}\right\}$ NMR spectrum of 2 . 
$\{\mathrm{K}[(\mathrm{HCDippN}) 2 \mathrm{BS}]\} 4[3]$

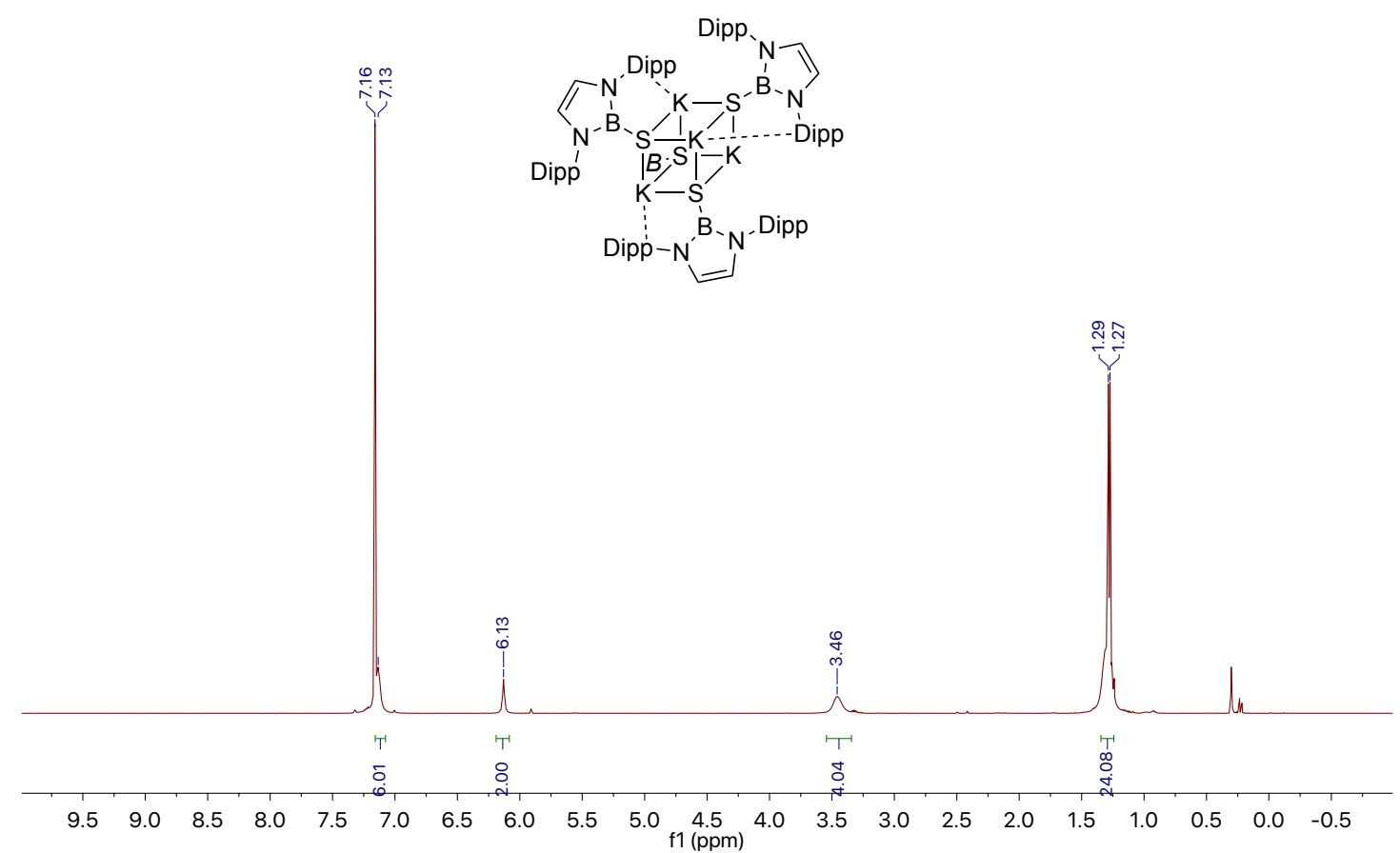

Figure S7. ${ }^{1} \mathrm{H}$ NMR spectrum of 3 .

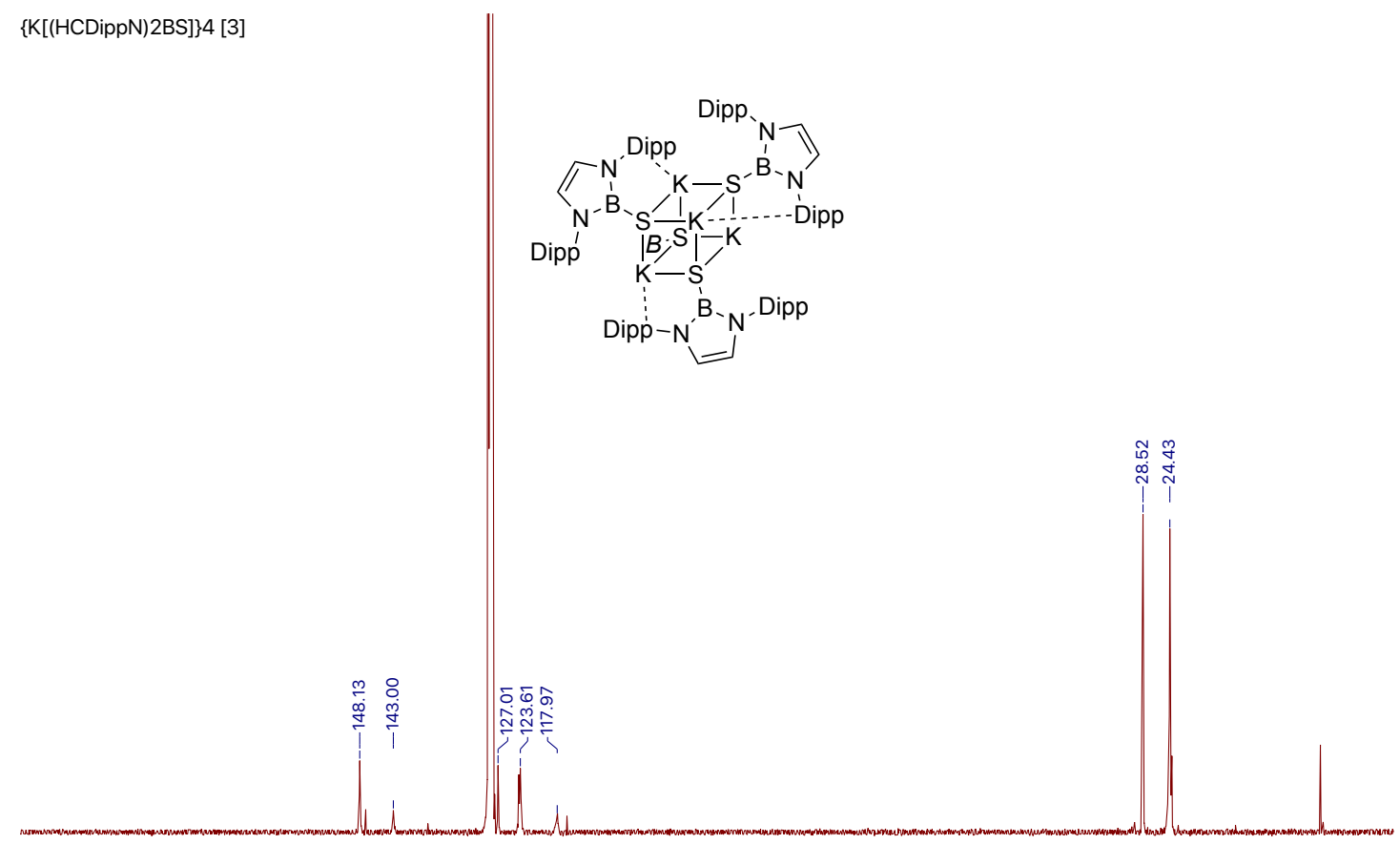

$\begin{array}{lllllllllllllllllll}190 & 180 & 170 & 160 & 150 & 140 & 130 & 120 & 110 & \underset{\mathrm{f} 1(\mathrm{ppm})}{100} & 80 & 70 & 60 & 50 & 40 & 30 & 20 & 10 & 0\end{array}$

Figure S8. ${ }^{13} \mathrm{C}\left\{{ }^{1} \mathrm{H}\right\}$ NMR spectrum of $\mathbf{3}$. 
$\{\mathrm{K}[(\mathrm{HCDippN}) 2 \mathrm{BS}]\} 4$ [3]

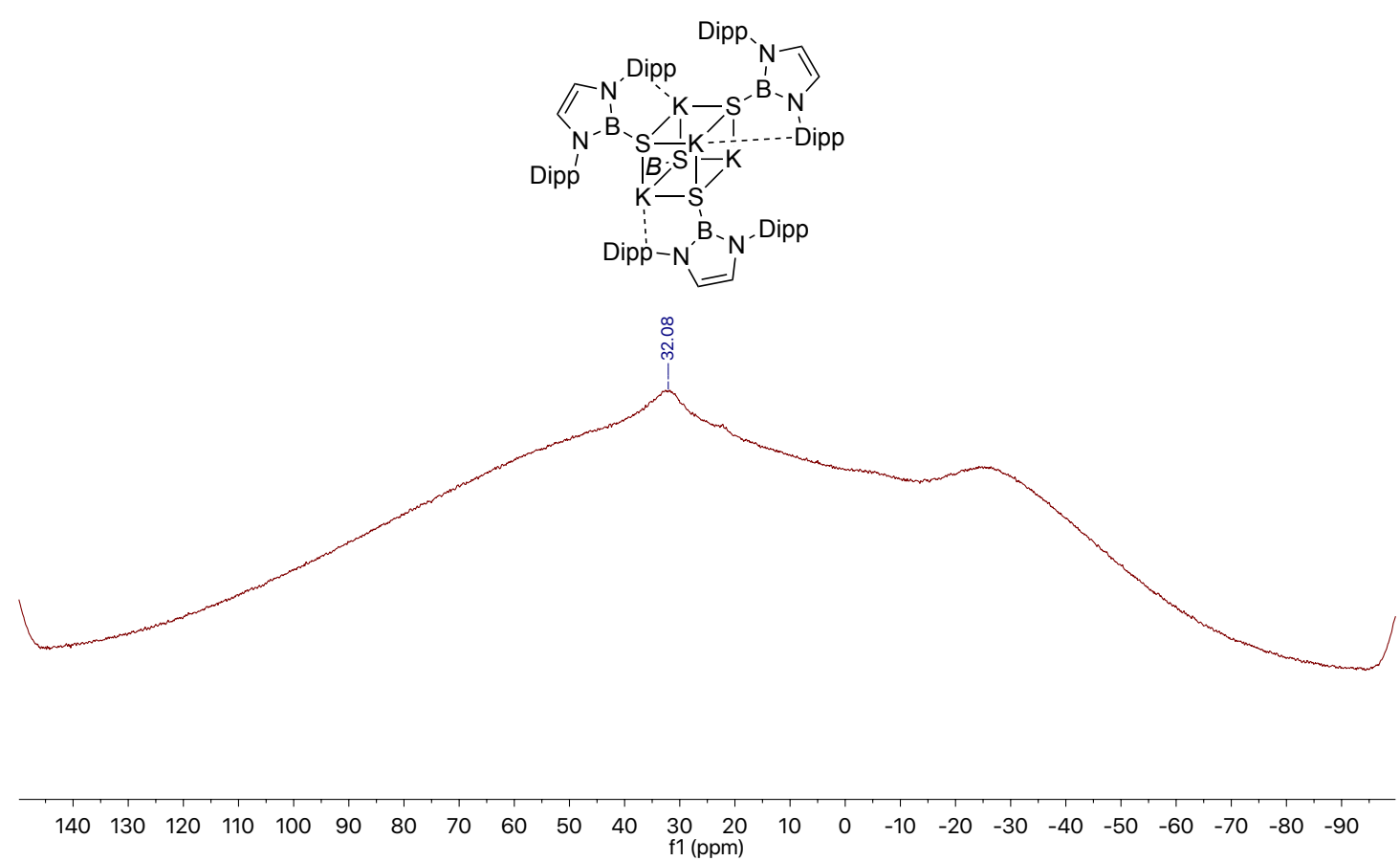

Figure S9. ${ }^{11} \mathrm{~B}\left\{{ }^{1} \mathrm{H}\right\}$ NMR spectrum of 3 . 


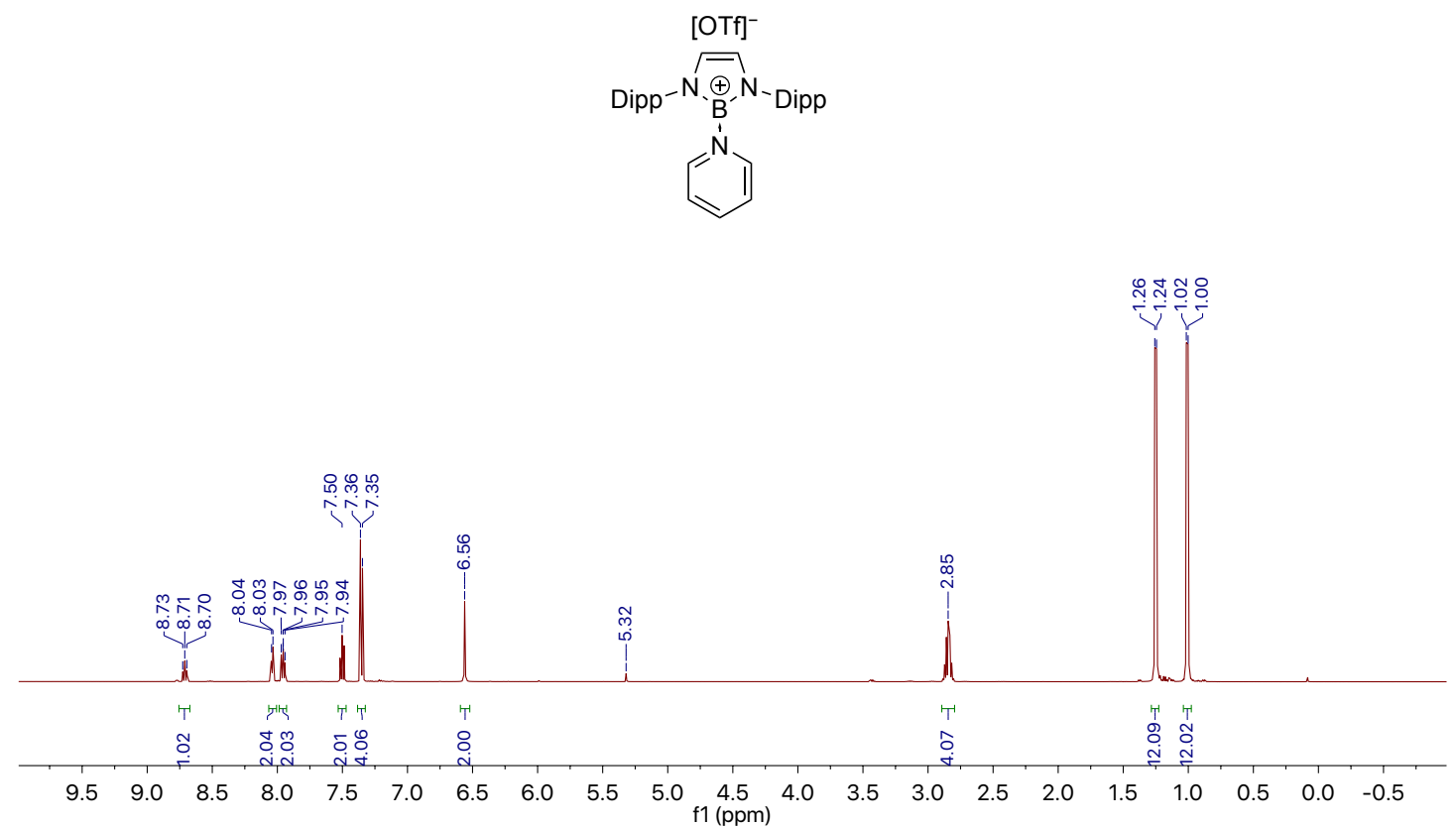

Figure S10. ${ }^{1} \mathrm{H}$ NMR spectrum of 4.

[(HCDippN)2B(NC5H5)][OTf] [4]

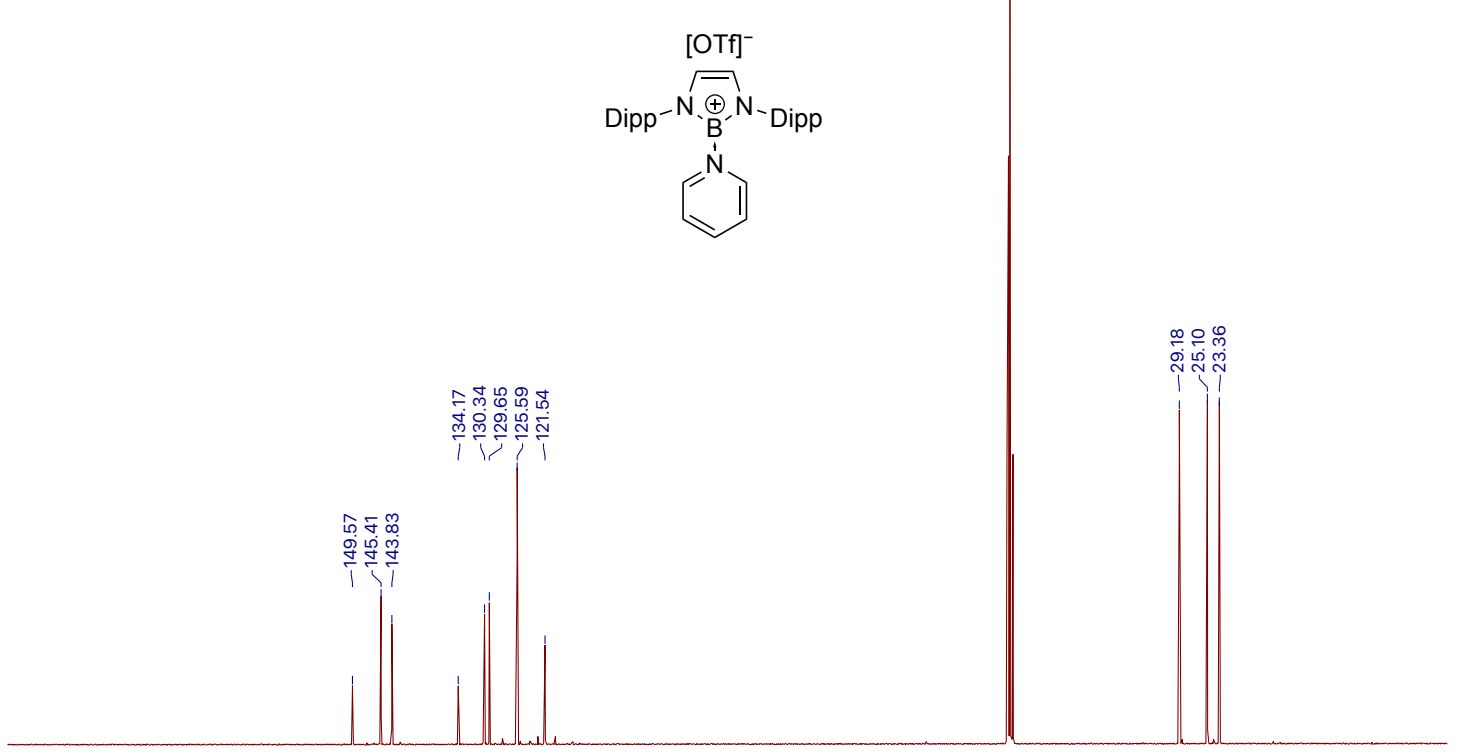

$\begin{array}{lllllllllllllllllll}190 & 180 & 170 & 160 & 150 & 140 & 130 & 120 & 110 & \begin{array}{c}100 \\ \mathrm{f} 1(\mathrm{ppm})\end{array} & 80 & 70 & 60 & 50 & 40 & 30 & 20 & 10 & 0\end{array}$

Figure S11. ${ }^{13} \mathrm{C}\left\{{ }^{1} \mathrm{H}\right\}$ NMR spectrum of 4 . 
[(HCDippN)2B(NC5H5)][OTf] [4]
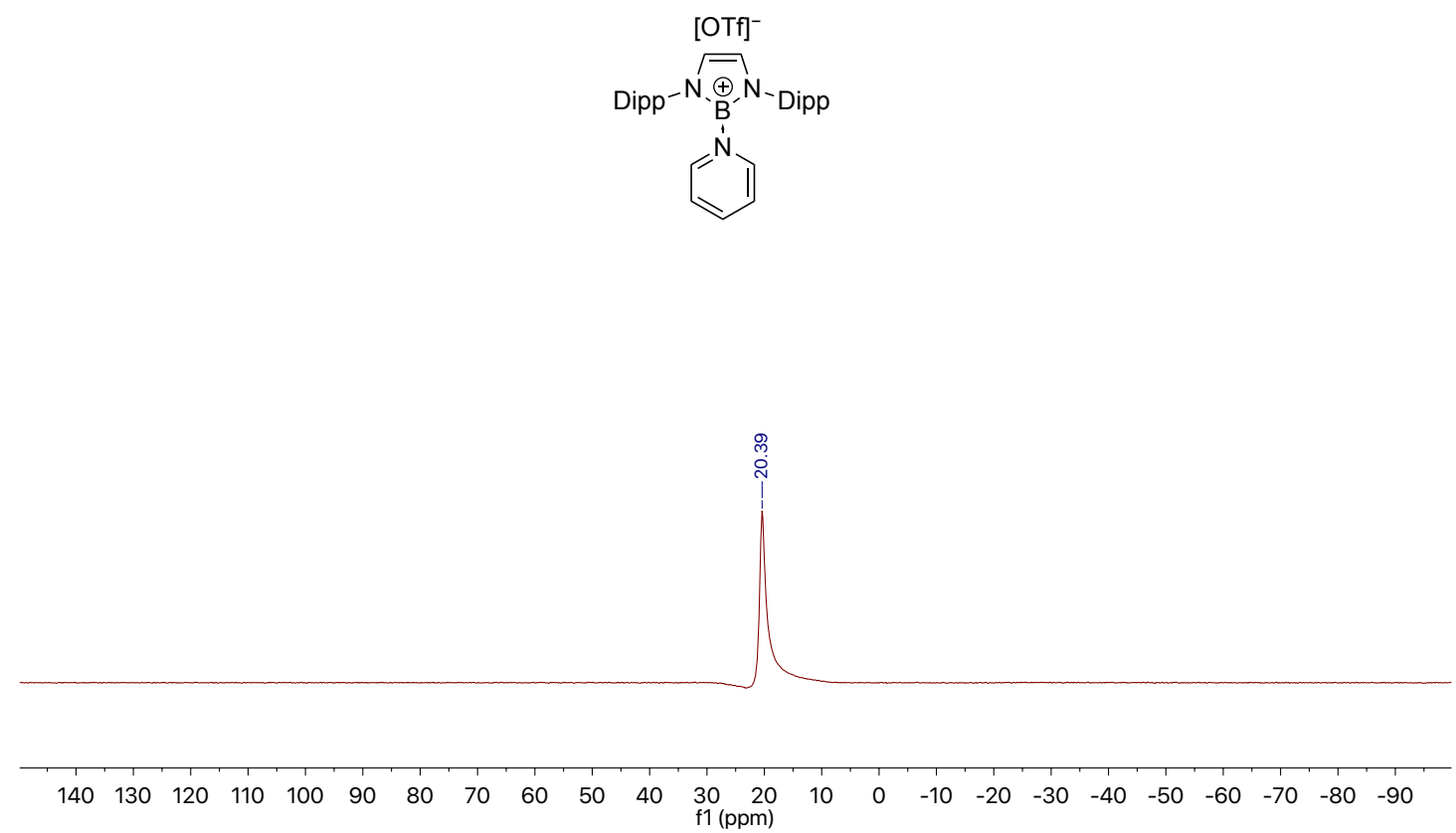

Figure S12. ${ }^{11} \mathrm{~B}\left\{{ }^{1} \mathrm{H}\right\}$ NMR spectrum of 4 .

[(HCDippN)2B(NC5H5)][OTf] [4]
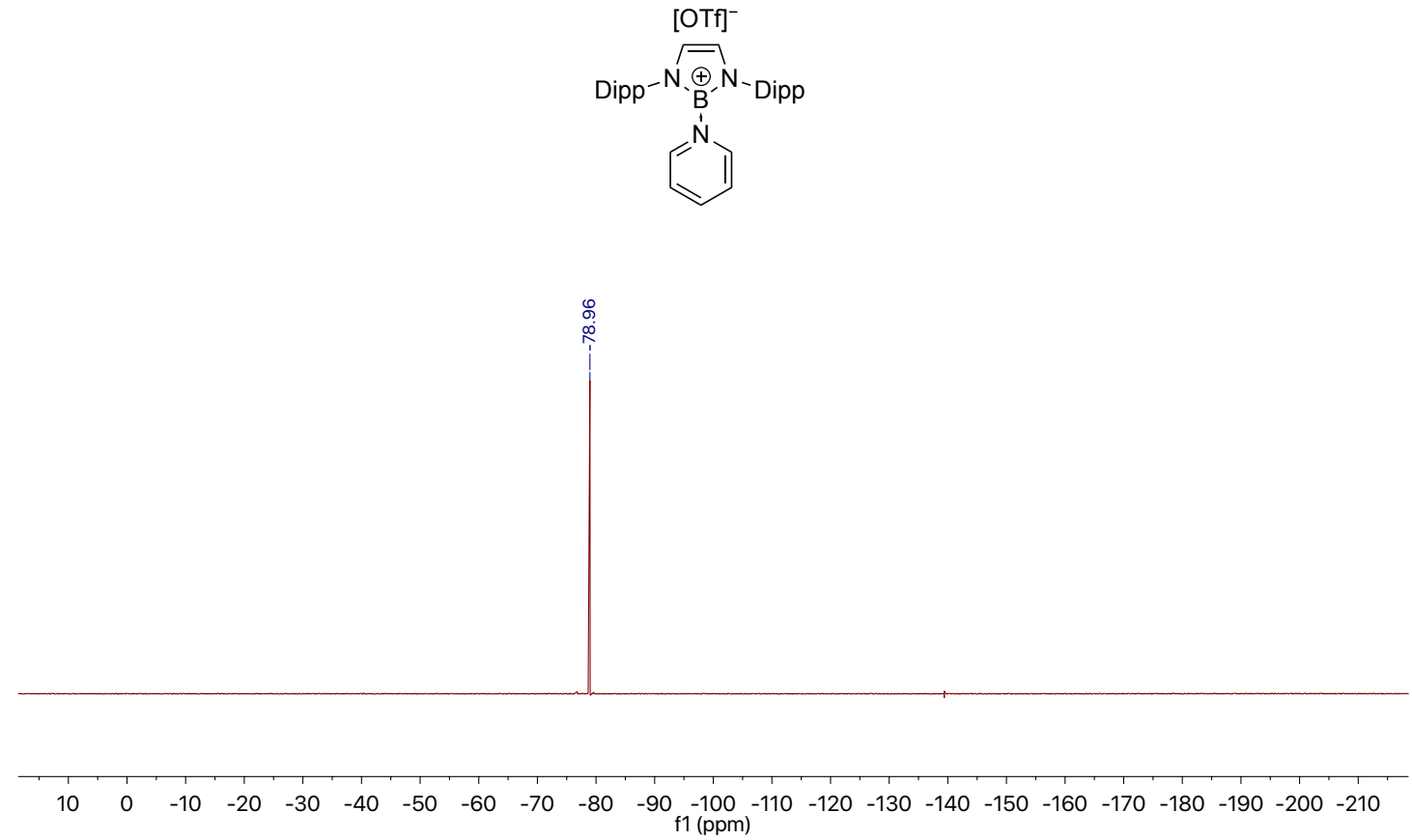

Figure S13. ${ }^{19} \mathrm{~F}\left\{{ }^{1} \mathrm{H}\right\}$ NMR spectrum of 4 . 


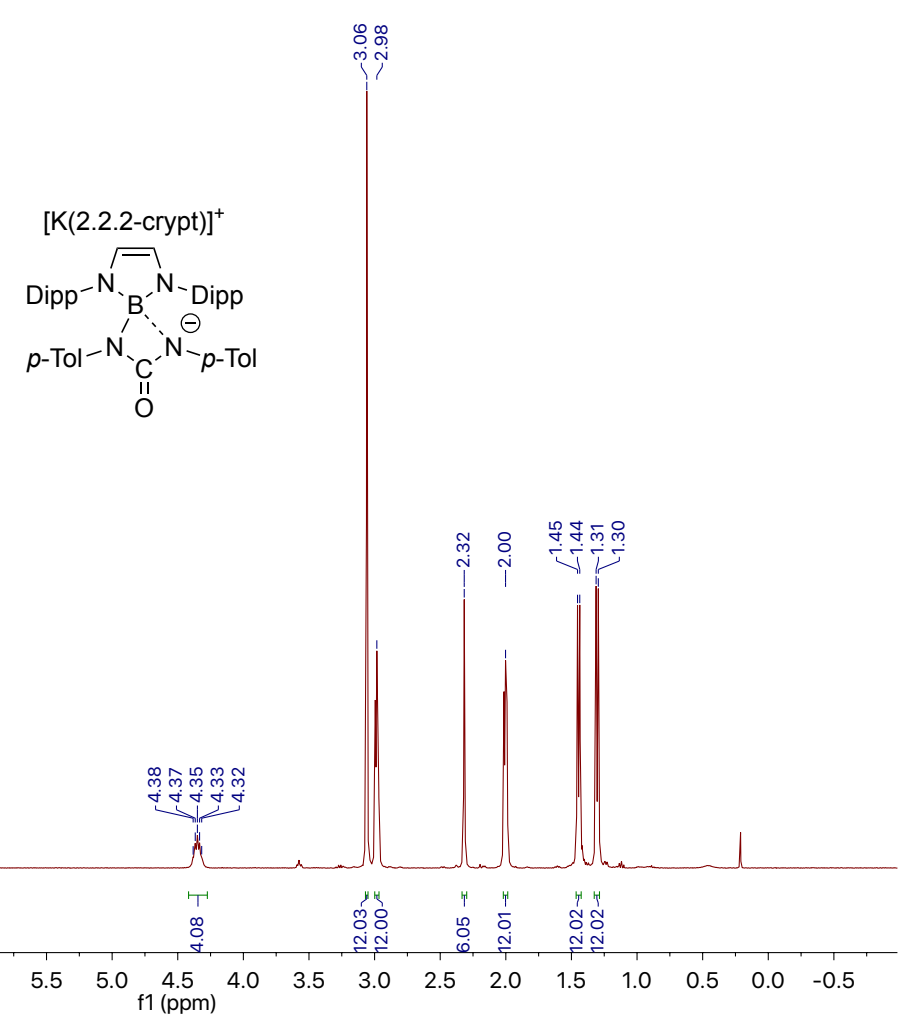

Figure S14. ${ }^{1} \mathrm{H}$ NMR spectrum of 5.

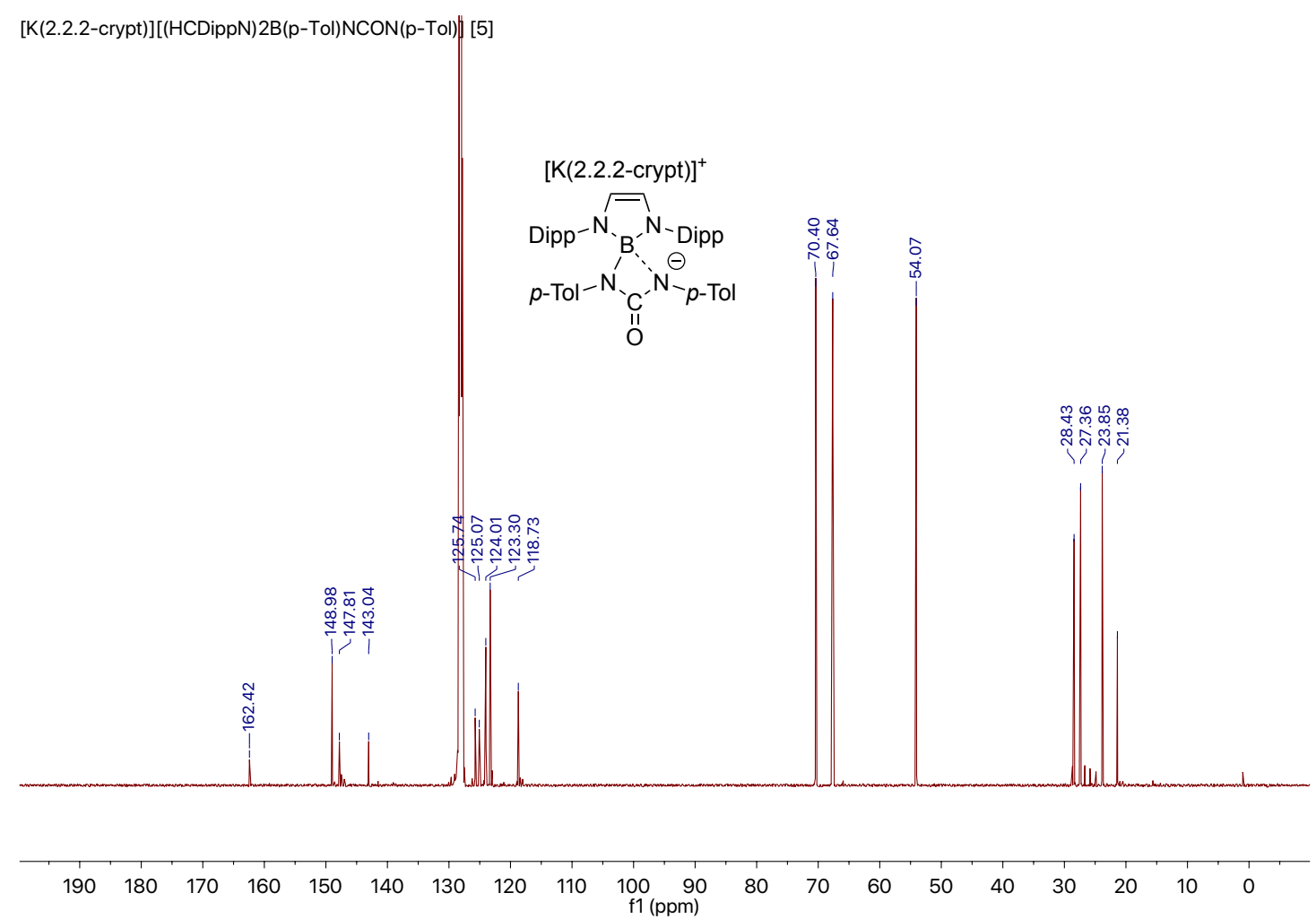

Figure S15. ${ }^{13} \mathrm{C}\left\{{ }^{1} \mathrm{H}\right\}$ NMR spectrum of 5 . 
[K(2.2.2-crypt)][(HCDippN)2B(p-Tol)NCON(p-Tol)] [5]

$$
[\mathrm{K}(2.2 .2-\mathrm{crypt})]^{+}
$$
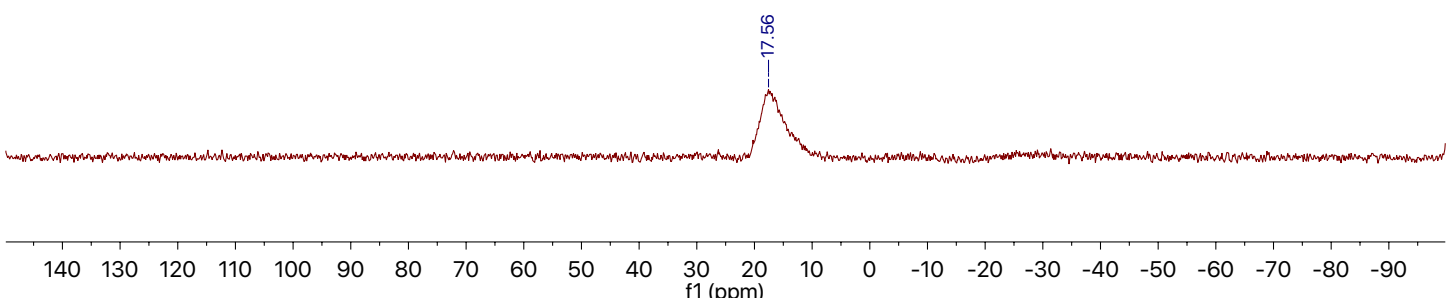

Figure S16. ${ }^{11} \mathrm{~B}\left\{{ }^{1} \mathrm{H}\right\}$ NMR spectrum of 5 . 


\section{4. $\quad$ X-ray crystallographic studies}

Crystal structure determinations:

Single-crystal X-ray diffraction data for 1-5 were collected at $150 \mathrm{~K}$ on Oxford Diffraction/Agilent SuperNova diffractometers with $\mathrm{Cu}-K \alpha(\lambda=1.54184 \AA)$ radiation equipped with nitrogen gas Oxford Cryosystems Cryostream unit. ${ }^{4}$ Raw frame data were reduced using CrysAlisPro. ${ }^{5}$ The structures were solved using SHELXT $^{6}$ and refined to convergence on $F^{2}$ and against all independent reflections by full-matrix least-squares using $\mathrm{SHELXL}^{7}$ in combination with the SHELXLE ${ }^{8}$ program. Distances and angles were calculated using the full covariance matrix. Restraints were used to maintain sensible geometries for the disordered groups and approximate the displacement parameters to typical values. Selected crystallographic data are summarized in the Table S1 and full details are given in the supplementary deposited CIF files (CCDC 1906845-1906849). These data can be obtained free of charge from the Cambridge Crystallographic Data Centre via http://www.ccdc.cam.ac.uk/data request/cif. 


\begin{tabular}{|c|c|c|c|c|c|}
\hline & 1 & 2 & 3 & 4 & 5 \\
\hline Formula & $\mathrm{C}_{94} \mathrm{H}_{150} \mathrm{~B}_{2} \mathrm{~K}_{2} \mathrm{~N}_{8} \mathrm{O}_{14}$ & $\mathrm{C}_{68} \mathrm{H}_{96} \mathrm{~B} \mathrm{~K} \mathrm{~N}_{4} \mathrm{O}_{6} \mathrm{~S}$ & $\mathrm{C}_{125} \mathrm{H}_{165} \mathrm{~B}_{4} \mathrm{~K}_{4} \mathrm{~N}_{8} \mathrm{~S}_{4}$ & $\mathrm{C}_{32} \mathrm{H}_{41} \mathrm{~B} \mathrm{~F}_{3} \mathrm{~N}_{3} \mathrm{O}_{3} \mathrm{~S}$ & $\mathrm{C}_{59} \mathrm{H}_{86} \mathrm{~B} \mathrm{~K} \mathrm{~N}_{6} \mathrm{O}_{7}$ \\
\hline $\mathrm{FW}_{\mathrm{W}}$ & 1716.03 & 1147.45 & 2107.52 & 615.55 & 1041.24 \\
\hline Cryst. System & Triclinic & Triclinic & Triclinic & Monoclinic & Monoclinic \\
\hline Space Group & P 1 & $\mathrm{P}-1$ & P -1 & P 21/c & P 21/c \\
\hline Wavelength/Å & 1.54184 & 1.54184 & 1.54184 & 1.54184 & 1.54184 \\
\hline$a / \AA$ & $13.8502(2)$ & $13.0099(3)$ & $15.5333(3)$ & $11.8476(2)$ & $12.24440(10)$ \\
\hline$b / \AA$ & $16.6927(3)$ & $15.9376(4)$ & $16.3399(3)$ & $13.6268(2)$ & $15.9465(2)$ \\
\hline$c / \AA$ & $23.0193(3)$ & $16.4118(4)$ & $27.9110(7)$ & $19.9995(3)$ & $30.3954(3)$ \\
\hline$\alpha /{ }^{\circ}$ & $90.2510(10)$ & $80.367(2)$ & $85.492(2)$ & 90 & 90 \\
\hline$\beta /{ }^{\circ}$ & $90.3870(10)$ & $86.833(2)$ & $89.509(2)$ & $94.4960(10)$ & $92.7400(10)$ \\
\hline$\gamma /{ }^{\circ}$ & $112.097(2)$ & $86.675(2)$ & $61.864(2)$ & 90 & 90 \\
\hline Volume $/ \AA^{3}$ & $4930.84(15)$ & $3345.71(14)$ & $6224.4(3)$ & $3218.88(9)$ & $5928.08(11)$ \\
\hline$Z$ & 2 & 2 & 2 & 4 & 4 \\
\hline Temp./K & $150(2)$ & $150(2)$ & $150(2)$ & $150(2)$ & $150(2)$ \\
\hline Refls. Collect. & 135248 & 47664 & 58648 & 18302 & 28293 \\
\hline $2 \theta_{\max }$ & 152.50 & 152.45 & 140.14 & 152.93 & 153.26 \\
\hline$R_{\text {int }}$ & 0.0478 & 0.0336 & 0.0411 & 0.0352 & 0.0263 \\
\hline Goodness of fit & 1.041 & 1.029 & 1.016 & 1.029 & 1.030 \\
\hline$R\left[F^{2}>2 \sigma\right], \mathrm{F}$ & 0.0551 & 0.0386 & 0.0680 & 0.0395 & 0.0422 \\
\hline$R_{w}$ (all data), $\mathrm{F}^{2}$ & 0.1516 & 0.1051 & 0.2101 & 0.1065 & 0.1156 \\
\hline
\end{tabular}




\section{Computational studies}

All reported computational work casrried out using Density Functional Theory, employing the Gaussian09 (Revision D.01) program package. ${ }^{9}$ Geometry optimizations were performed in the gas phase, with PBE1PBE exchange-correlation functional ${ }^{10}$ and def-TZVP basis set. ${ }^{11}$ Grimme's empirical dispersion correction (DFT-D3) was also used. ${ }^{12}$ The nature of stationary points found (minima) was confirmed by full frequency calculations. Natural bond orbital (NBO) analyses were performed using NBO 3.1 as implemented in Gaussian09. ${ }^{13}$

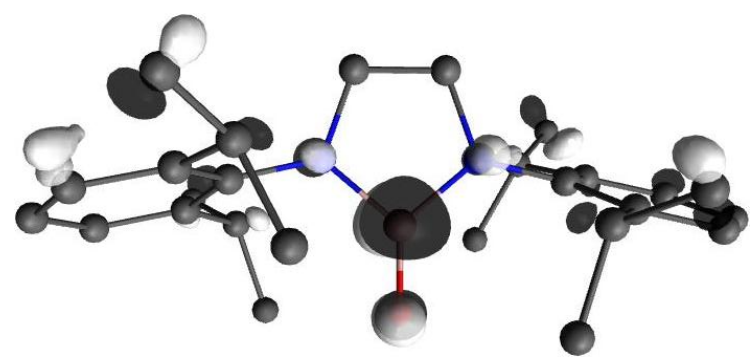

Figure S17. $\mathrm{LUMO}+31$ of 1 showing the $\mathrm{B}=\mathrm{O} \pi *$ orbital.

\section{xyz-coordinates of optimized structures:}

$\begin{array}{lrrr}\left.\text { [(HCDippN })_{2} \mathrm{BO}\right] & & \\ 66 & & & \\ \mathrm{~B} & 0.000100 & 0.000100 & -0.425200 \\ \mathrm{~N} & 1.143300 & -0.084700 & 0.551200 \\ \mathrm{C} & 0.673200 & -0.041300 & 1.858800 \\ \mathrm{H} & 1.340500 & -0.077200 & 2.707800 \\ \mathrm{C} & -0.673400 & 0.041700 & 1.858700 \\ \mathrm{H} & -1.340700 & 0.077600 & 2.707600 \\ \mathrm{~N} & -1.143300 & 0.085000 & 0.551000 \\ \mathrm{O} & 0.000300 & 0.000100 & -1.714200 \\ \mathrm{C} & 2.513400 & -0.059500 & 0.249800 \\ \mathrm{C} & 3.155800 & -1.229600 & -0.181400 \\ \mathrm{C} & 4.515000 & -1.176000 & -0.481100 \\ \mathrm{H} & 5.022500 & -2.073400 & -0.820500 \\ \mathrm{C} & 5.228400 & 0.003400 & -0.353100 \\ \mathrm{H} & 6.289200 & 0.026100 & -0.583400 \\ \mathrm{C} & 4.582200 & 1.159900 & 0.058800 \\ \mathrm{H} & 5.142200 & 2.085800 & 0.135500 \\ \mathrm{C} & 3.222800 & 1.150300 & 0.349800 \\ \mathrm{C} & 2.366500 & -2.504100 & -0.363700 \\ \mathrm{H} & 1.450800 & -2.384900 & 0.221400 \\ \mathrm{C} & 3.098800 & -3.739700 & 0.147200 \\ \mathrm{H} & 3.986800 & -3.966100 & -0.451700 \\ \mathrm{H} & 2.441600 & -4.612700 & 0.091200 \\ \mathrm{H} & 3.416400 & -3.616800 & 1.186400 \\ \mathrm{C} & 1.952700 & -2.659800 & -1.827200 \\ \mathrm{H} & 1.361900 & -1.793400 & -2.142800 \\ \mathrm{H} & 1.347300 & -3.563300 & -1.958100 \\ \mathrm{H} & 2.838300 & -2.747600 & -2.467200 \\ \mathrm{C} & 2.466300 & 2.421800 & 0.660700 \\ \mathrm{H} & 1.643200 & 2.153600 & 1.328700 \\ \mathrm{C} & 1.845700 & 2.965600 & -0.628600 \\ \mathrm{H} & 2.632200 & 3.289500 & -1.318900 \\ \mathrm{H} & 1.203800 & 3.826700 & -0.413900 \\ \mathrm{H} & 1.247900 & 2.200900 & -1.131500 \\ \mathrm{C} & 3.304300 & 3.485800 & 1.356100 \\ \mathrm{H} & 3.782400 & 3.096300 & 2.259400 \\ \mathrm{H} & 2.671100 & 4.330200 & 1.643100 \\ \mathrm{H} & 4.088500 & 3.880100 & 0.702000 \\ \mathrm{C} & -2.513400 & 0.059600 & 0.249600 \\ & & & \\ \end{array}$




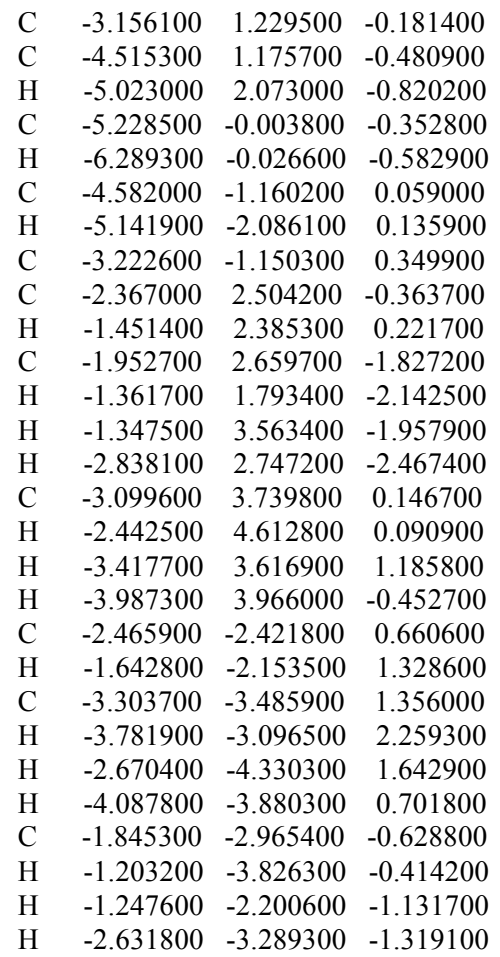

\begin{tabular}{|c|c|c|c|}
\hline $\mathrm{C}$ & 0.649100 & 0.122100 & -1.864800 \\
\hline $\mathrm{H}$ & 1.319200 & 0.196600 & -2.708000 \\
\hline $\mathrm{N}$ & 1.115900 & 0.073000 & -0.557400 \\
\hline $\mathrm{S}$ & -0.000100 & -0.120500 & 2.142900 \\
\hline B & -0.009100 & -0.033500 & 0.387200 \\
\hline $\mathrm{C}$ & 2.492600 & 0.087800 & -0.257500 \\
\hline $\mathrm{N}$ & -1.150000 & -0.048100 & -0.547900 \\
\hline $\mathrm{C}$ & -0.698000 & 0.057100 & -1.859300 \\
\hline $\mathrm{H}$ & -1.378400 & 0.066600 & -2.697600 \\
\hline $\mathrm{C}$ & 3.226500 & -1.105400 & -0.345300 \\
\hline $\mathrm{C}$ & 4.586100 & -1.074400 & -0.055300 \\
\hline $\mathrm{H}$ & 5.167700 & -1.988200 & -0.114100 \\
\hline $\mathrm{C}$ & 5.205600 & 0.105600 & 0.327600 \\
\hline $\mathrm{H}$ & 6.266800 & 0.112600 & 0.556500 \\
\hline $\mathrm{C}$ & 3.104000 & 1.283400 & 0.138000 \\
\hline $\mathrm{C}$ & 4.466100 & 1.271100 & 0.427000 \\
\hline $\mathrm{H}$ & 4.950900 & 2.189800 & 0.742600 \\
\hline $\mathrm{C}$ & 2.305700 & 2.558300 & 0.277200 \\
\hline $\mathrm{H}$ & 1.293800 & 2.337300 & -0.067700 \\
\hline $\mathrm{C}$ & 2.204800 & 2.973200 & 1.742400 \\
\hline $\mathrm{H}$ & 1.744200 & 2.167100 & 2.319400 \\
\hline $\mathrm{H}$ & 1.584200 & 3.869600 & 1.842500 \\
\hline $\mathrm{H}$ & 3.193800 & 3.195800 & 2.158100 \\
\hline $\mathrm{C}$ & 2.868600 & 3.679300 & -0.591900 \\
\hline $\mathrm{H}$ & 3.878800 & 3.962500 & -0.279400 \\
\hline $\mathrm{H}$ & 2.237600 & 4.570300 & -0.517400 \\
\hline $\mathrm{H}$ & 2.914600 & 3.380000 & -1.642500 \\
\hline $\mathrm{C}$ & 2.520100 & -2.404700 & -0.661700 \\
\hline $\mathrm{H}$ & 1.616300 & -2.152300 & -1.221200 \\
\hline $\mathrm{C}$ & 2.074400 & -3.077500 & 0.637300 \\
\hline $\mathrm{H}$ & 1.488700 & -3.977400 & 0.420700 \\
\hline $\mathrm{H}$ & 1.463900 & -2.397000 & 1.238900 \\
\hline $\mathrm{H}$ & 2.945500 & -3.370900 & 1.233000 \\
\hline $\mathrm{C}$ & 3.349700 & -3.351700 & -1.519900 \\
\hline $\mathrm{H}$ & 2.750100 & -4.222300 & -1.801000 \\
\hline $\mathrm{H}$ & 4.227800 & -3.724600 & -0.983800 \\
\hline $\mathrm{H}$ & 3.695500 & -2.864900 & -2.436100 \\
\hline $\mathrm{C}$ & -2.525900 & -0.068600 & -0.243600 \\
\hline $\mathrm{C}$ & -3.117300 & -1.259400 & 0.204000 \\
\hline $\mathrm{C}$ & -4.485900 & -1.263100 & 0.454300 \\
\hline
\end{tabular}




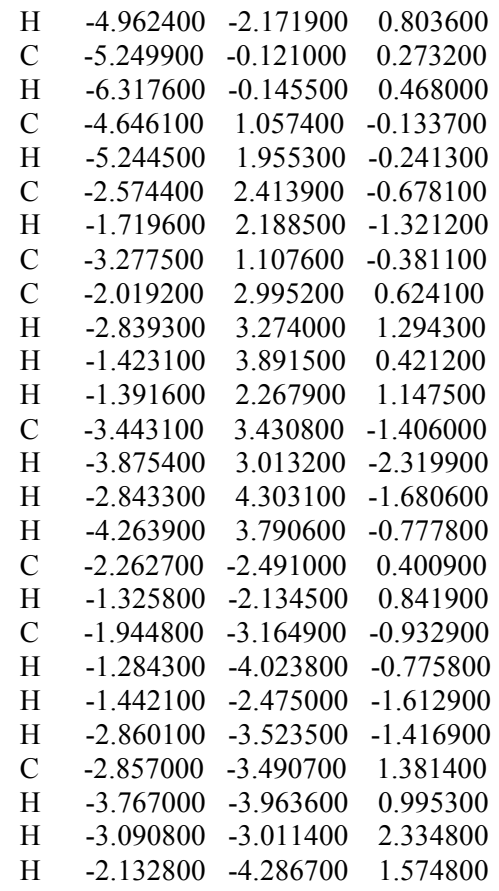

$\begin{array}{lrrr}\text { (HCDippN })_{2} \mathrm{CO} & & \\ 66 & & & \\ \mathrm{~N} & 1.090300 & -0.051700 & 0.649400 \\ \mathrm{C} & 0.671100 & -0.033000 & 1.973300 \\ \mathrm{H} & 1.375000 & -0.067100 & 2.786600 \\ \mathrm{C} & -0.671200 & 0.033000 & 1.973200 \\ \mathrm{H} & -1.375300 & 0.067000 & 2.786500 \\ \mathrm{~N} & -1.090400 & 0.051700 & 0.649200 \\ \mathrm{O} & 0.000100 & 0.000000 & -1.420000 \\ \mathrm{C} & 2.442200 & -0.086900 & 0.209500 \\ \mathrm{C} & 2.991800 & -1.306200 & -0.192300 \\ \mathrm{C} & 4.319300 & -1.310700 & -0.611400 \\ \mathrm{H} & 4.772900 & -2.241000 & -0.934400 \\ \mathrm{C} & 5.064400 & -0.145400 & -0.625500 \\ \mathrm{H} & 6.098200 & -0.168900 & -0.952600 \\ \mathrm{C} & 4.494300 & 1.054100 & -0.228500 \\ \mathrm{H} & 5.086200 & 1.961200 & -0.253900 \\ \mathrm{C} & 3.169900 & 1.109200 & 0.188800 \\ \mathrm{C} & 2.177400 & -2.578200 & -0.220000 \\ \mathrm{H} & 1.226700 & -2.374100 & 0.278300 \\ \mathrm{C} & 2.858000 & -3.714300 & 0.537300 \\ \mathrm{H} & 3.798100 & -4.007500 & 0.062800 \\ \mathrm{H} & 2.211600 & -4.595600 & 0.554800 \\ \mathrm{H} & 3.077400 & -3.431000 & 1.569500 \\ \mathrm{C} & 1.868300 & -2.974700 & -1.663100 \\ \mathrm{H} & 1.349700 & -2.166500 & -2.181700 \\ \mathrm{H} & 1.235800 & -3.866200 & -1.687600 \\ \mathrm{H} & 2.789200 & -3.200200 & -2.208600 \\ \mathrm{C} & 2.506600 & 2.424000 & 0.532000 \\ \mathrm{H} & 1.679400 & 2.209300 & 1.214100 \\ \mathrm{C} & 1.910200 & 3.043900 & -0.733500 \\ \mathrm{H} & 2.704500 & 3.310300 & -1.436700 \\ \mathrm{H} & 1.353900 & 3.953500 & -0.489500 \\ \mathrm{H} & 1.237300 & 2.349300 & -1.239800 \\ \mathrm{C} & 3.433800 & 3.405500 & 1.237100 \\ \mathrm{H} & 3.899500 & 2.958100 & 2.118400 \\ \mathrm{H} & 2.869500 & 4.284000 & 1.559200 \\ \mathrm{H} & 4.229900 & 3.760200 & 0.577200 \\ \mathrm{C} & -2.442200 & 0.086900 & 0.209300 \\ \mathrm{C} & -2.991800 & 1.306200 & -0.192500 \\ \mathrm{C} & -4.319300 & 1.310700 & -0.611800 \\ \mathrm{H} & -4.772800 & 2.241000 & -0.934800 \\ \mathrm{C} & -5.064300 & 0.145400 & -0.626000 \\ \mathrm{H} & -6.098100 & 0.168900 & -0.953200 \\ & & & \end{array}$




$\begin{array}{lrrr}\mathrm{C} & -4.494300 & -1.054200 & -0.229000 \\ \mathrm{H} & -5.086100 & -1.961200 & -0.254400 \\ \mathrm{C} & -3.169900 & -1.109200 & 0.188500 \\ \mathrm{C} & -2.177400 & 2.578300 & -0.220100 \\ \mathrm{H} & -1.226800 & 2.374100 & 0.278200 \\ \mathrm{C} & -1.868200 & 2.974800 & -1.663200 \\ \mathrm{H} & -1.349500 & 2.166600 & -2.181700 \\ \mathrm{H} & -1.235700 & 3.866300 & -1.687600 \\ \mathrm{H} & -2.789000 & 3.200300 & -2.208800 \\ \mathrm{C} & -2.858100 & 3.714300 & 0.537100 \\ \mathrm{H} & -2.211800 & 4.595600 & 0.554800 \\ \mathrm{H} & -3.077600 & 3.431000 & 1.569300 \\ \mathrm{H} & -3.798200 & 4.007400 & 0.062500 \\ \mathrm{C} & -2.506600 & -2.424000 & 0.531700 \\ \mathrm{H} & -1.679500 & -2.209300 & 1.213800 \\ \mathrm{C} & -3.433900 & -3.405600 & 1.236700 \\ \mathrm{H} & -3.899700 & -2.958100 & 2.118000 \\ \mathrm{H} & -2.869600 & -4.284000 & 1.558800 \\ \mathrm{H} & -4.229900 & -3.760300 & 0.576700 \\ \mathrm{C} & -1.910000 & -3.043800 & -0.733800 \\ \mathrm{H} & -1.353800 & -3.953500 & -0.489700 \\ \mathrm{H} & -1.237100 & -2.349200 & -1.240000 \\ \mathrm{H} & -2.704300 & -3.310300 & -1.437100 \\ \mathrm{C} & 0.000000 & 0.000000 & -0.203200\end{array}$


(1) Loh, Y. K.; Ying, L.; Fuentes, M. A.; Do, D. C. H.; Aldridge, S. Angew. Chem., Int. Ed. 2019, 58, 4847-4851.

(2) Segawa, Y.; Suzuki, Y.; Yamashita, M.; Nozaki, N. J. Am. Chem. Soc. 2008, 130, 16069-16079.

(3) ACD-S: Specs Research Laboratory (Spectral data were obtained from Advanced Chemistry Development, Inc.).

(4) Cosier, J.; Glazer, A. M. A nitrogen-gas-stream cryostat for general X-ray diffrcaction studies. J. Appl. Cryst. 1986, 19, 105-107.

(5) CrysAlisPro, Agilent Technologies, Version 1.171.35.8.

(6) Sheldrick, G. M. SHELXT - Integrated space-group and crystal structure determination. Acta Cryst. Sect. A 2015, 71, 3-8.

(7) Sheldrick, G. M. Acta Cryst. Sect. A 2008, 64, 112-122.

(8) Hübschle, C. B.; Sheldrick, G. M.; Dittrich, B. ShelXle: a Qt graphical user interface for SHELXL. J. Appl. Cryst. 2011, 44, 1281-1284.

(9) Frisch, M. J.; Trucks, G. W.; Schlegel, H. B.; Scuseria, G. E.; Robb, M. A.; Cheeseman, J. R.; Scalmani, G.; Barone, V.; Mennucci, B.; Petersson, G. A.; Nakatsuji, H.; Li, X.; Caricato, M.; Li, X.; Hratchian, H. P.; Izmaylov, A. F.; Bloino, J.; Zheng, G.; Sonnenberg, J. L.; Hada, M.; Ehara, M.; Toyota, K.; Fukuda, R.; Hasegawa, J.; Ishida, M.; Nakajima, T.; Honda, Y.; Kitao, O.; Nakai, H.; Vreven, T.; Montgomery, J. A., Jr.; Peralta, J. E.; Ogliaro, F.; Bearpark, M. J.; Heyd, J. J.; Brothers, E. N.; Kudin, K. N.; Staroverov, V. N.; Kobayashi, R.; Normand, J.; Raghavachari, K.; Rendell, A. P.; Burant, J. C.; Iyengar, S. S.; Tomasi, J.; Cossi, M.; Rega, N.; Millam, J. M.; Klene, M.; Adamo, C.; Jaramillo, J.; Gomperts, R.; Stratmannm R. E.; Yazyev, O; Austin, A.J.; Cammi, R.; Pomelli, C.; Ochterski, J. W.; Martin, R. L.; Morokuma, K.; Zakrzewski, V. G.; Voth, G. A.; Salvador, P; Dannenberg, J. J.; Dapprich, S.; Daniels, A. D.; Farkas, O.; Foresman, J. B.; Ortiz, J. V.; Cioslowski, J.; Fox, D. J. Gaussian 09, Revision D.01, Gaussian, Inc., Wallingford, CT 2009.

(10)(a) Ernzerhof, M.; Scuseria, G. E. J. Chem. Phys. 1999, 110, 5029-5036. (b) Adamo, C.; Barone, V. J. Chem. Phys. 1999, 110, 6158-6170. (c) Perdew, J. P.; Burke, K.; Ernzerhof, M. Phys. Rev. Lett. 1997, 78, 1396. (d) Perdew, J. P.; Burke, K.; Ernzerhof, M. Phys. Rev. Lett. 1996, 77, 3865-3868. (e) Perdew, J. P.; Ernzerhof, M.; Burke, K. J. Chem. Phys. 1996, 105, 9982-9985.

(11) Schaefer, A.; Huber, C; Ahlrichs, R. J. Chem. Phys. 1994, 100, 5829-5835.

(12) (a) Grimme, S.; Antony, J.; Ehrlich, S.; Krieg, H. J. Chem. Phys. 2010, 132, 154104-154119. (b) Grimme, S.; Ehrlich, S.; Goerigk, L. J. Comput. Chem. 2011, 32, 1456-1465.

(13) Glendening, E. D.; Reed, A. E.; Carpenter, J. E.; Weinhold,F. NBO Version 3.1. 